\title{
A WELL-CONDITIONED INTEGRAL EQUATION FOR ITERATIVE SOLUTION OF SCATTERING PROBLEMS WITH A VARIABLE LEONTOVITCH BOUNDARY CONDITION
}

\author{
SÉBASTIEN PERnet ${ }^{1}$
}

\begin{abstract}
The construction of a well-conditioned integral equation for iterative solution of scattering problems with a variable Leontovitch boundary condition is proposed. A suitable parametrix is obtained by using a new unknown and an approximation of the transparency condition. We prove the well-posedness of the equation for any wavenumber. Finally, some numerical comparisons with well-tried method prove the efficiency of the new formulation.
\end{abstract}

Mathematics Subject Classification. 65R20, 15A12, 65N38, 65F10, 65Z05.

Received September 15, 2007. Revised June 22, 2009.

Published online March 17, 2010.

\section{IntRoduction}

This paper deals with the solution of electromagnetic scattering problems by an obstacle whose surface is covered by thin layers of imperfectly conductor materials. This type of materials is generally taken into account by imposing an impedance boundary condition like the Leontovitch condition [21] on the surface of the object. It was recognized that this type of boundary condition can be extensively used to get a tractable problem in numerous complex situations. A first example can be found in radar applications: objects are often partially coated by a thin dielectric layer to reduce the radar cross section of scattering waves; in this case, the direct scattering problem amounts to a mixed boundary value problem with Maxwell's equations posed on an unbounded domain and where on the coated part of the boundary the electromagnetic field satisfies an impedance boundary condition while on the remaining part of the boundary the tangential component of the total electric field vanishes. Another domain of application is the use of this condition as an absorbing boundary condition to limit the computational domain of a finite elements method [25]. This condition plays also a major role in the domains decomposition method for Maxwell's equations [5,13,31]. Thus, it appears crucial to have efficient numerical methods well suited for such boundary conditions.

In the frequency domain, the Boundary Integral Methods (BIM) are a very attractive tool to solve electromagnetic problems. Numerous studies have been conducted about the choice and resolution of an integral equation for many years. Among significant accomplishments (this list is not exhaustive): the development of equations which are well-posed for any frequency [22,24], the development of the Fast Multipole Method (FMM) which allows to reduce the matrix-vector product to $O(N \log (N))$ complexity and consequently gives the possibility to treat big problems (several millions degrees of freedom) [29] and more recently the emergence of "naturally"

Keywords and phrases. Electromagnetic scattering, boundary integral equations, impedance boundary condition, preconditioner.

1 CERFACS, 42 avenue Gaspard Coriolis, 31057 Toulouse Cedex 01, France. sebastien.pernet@cerfacs.fr 
well-conditioned formulations which are derived by finding a analytical preconditioner or parametrix (in sense of the pseudo-differential calculus) of the underlying integral operator $[1,12,17,30]$. The latter technique allows to obtain Fredholm integral equations of second kind which decompose under the form "Identity + C" where C is a compact operator and which lead to good rates of convergence when an iterative solver is used to solved the linear system. In particular, $[1,17]$ have shown that the convergence rate is wavenumber $(k)$ and mesh-size $(h)$ independent (for the smooth surfaces). That is why this technique is attractive, compared to the classical algebraic preconditioners like SPAI (SParse Approximate Inverse) where the $k$ and $h$-dependences are not obvious to take into account. The aim of this paper is to propose a well-conditioned integral equation to solve for the scattering problems with a variable Leontovitch boundary condition. This construction is based on an adequate parametrix which is obtained by using an approximation of the transparent condition $[2,18]$. The formulation is an extension to the impedance problems of this one proposed in [18] with furthermore first numerical results.

The outline of the paper is as follows. In Section 2, we present the mathematical model and the basic tools to derive the integral equations in electromagnetism. Section 3 is devoted to the construction of the wellconditioned integral equation. In Section 4, we prove that this equation corresponds to a compact perturbation of the identity operator and is one-to-one when the impedance operator and the surface are smooth. These last assumptions are necessary to use the pseudo-differential calculus. In Section 5, we present the discretization and the resolution of the integral formulation. Finally, in Section 6, some numerical test-cases allow us to show that we obtain good convergences rates for constant and piecewise constant impedance operators. Moreover, some comparisons with other integral method allow us to see that the proposed formulation is also competitive in terms of accuracy.

\section{The SCATTERING PROBlem AND INTEGRAL REPRESENTATION}

Let $\Omega^{-}$be a Lipschitz polyhedron with a boundary $\Gamma$ which is assumed to be simply connected and connected. The open complement of $\Omega^{-}$in $\mathbb{R}^{3}$ is $\Omega^{+}$. Vector $\mathbf{n}$ denotes the unit normal to $\Gamma$ pointing into the exterior domain $\Omega^{+}$of $\Omega^{-}$. The problem is to find the scattering electromagnetic fields $\mathbf{E}$ and $\mathbf{H}$ solution to the Maxwell system

$$
\left\{\begin{array}{l}
\operatorname{curl} \mathbf{E}-i k Z_{0} \mathbf{H}=0 \text { in } \Omega^{+}, \\
\operatorname{curl} \mathbf{H}+i k Z_{0}^{-1} \mathbf{E}=0 \text { in } \Omega^{+}
\end{array}\right.
$$

completed with both the Silver-Müller radiation condition at infinity

$$
\lim _{|x| \rightarrow \infty}|x|\left(\mathbf{E}(x)+Z_{0} \frac{x}{|x|} \times \mathbf{H}(x)\right)=0
$$

and the boundary conditions on the surface $\Gamma$

$$
\mathbf{n} \times\left(\mathbf{E}_{\mid \Gamma} \times \mathbf{n}\right)-Z_{0} \eta\left(\mathbf{n} \times \mathbf{H}_{\mid \Gamma}\right)=\mathbf{g}
$$

where $k>0$ is the wavenumber, $Z_{0}$ is the intrinsic impedance of the vacuum, $\mathbf{g}$ is a data and $\eta(x)$ is an impedance function. The variations of $\eta(x)$ allows us to take into account the presence of different materials on the surface $\Gamma$ of the obstacle.

We have the following existence and the uniqueness results (see [8] for a proof and [25] for details on this kind of techniques):

Theorem 2.1. We assume that:

- $\Omega^{-}$is a connected Lipschitz polyhedral domain.

- $\mathbf{g} \in \mathbf{H}_{T}^{0}(\Gamma)$.

- $\eta \in L^{\infty}(\Gamma)$ and is assumed to be a strictly-positive real-valued function.

Then the exterior mixed boundary value problem (2.1)-(2.2)-(2.3) has a unique solution which belongs to the space $X_{\mathrm{loc}}\left(\Omega^{+}, \Gamma\right):=\left\{\mathbf{u} \in \mathbf{H}_{\mathrm{loc}}\left(\operatorname{curl}, \Omega^{+}\right): \mathbf{n} \times \mathbf{u}_{\mid \Gamma} \in \mathbf{L}_{T}^{2}(\Gamma)\right\}$, where $\mathbf{L}_{T}^{2}(\Gamma)=\mathbf{H}_{T}^{0}(\Gamma)=\left\{\mathbf{u} \in\left[L^{2}(\Gamma)\right]^{3}: \mathbf{u} \cdot \mathbf{n}=0\right\}$. 
Remark 2.2. In what follows, we assume that the assumptions of Theorem 2.1 are verified. Moreover, the impedance function $\eta(x)$ is assumed to be piecewise constant.

Now, we are presenting some material from the classical scattering theory. First, we recall some functional spaces which allow to correctly define the trace and integral operators in the context of Lipschitz polyhedral domains [7]: the tangential trace operator $\gamma_{t}$ defined by:

$$
\begin{array}{ccc}
\gamma_{t}: \mathbf{H}(\operatorname{curl}, \Omega) & \rightarrow & \mathbf{H}_{\times}^{-1 / 2}\left(\operatorname{div}_{\Gamma}, \Gamma\right) \\
\mathbf{u} & \mapsto & \mathbf{n} \times \mathbf{u}
\end{array}
$$

is continuous, surjective and possesses a right inverse where $\mathbf{H}_{\times}^{-1 / 2}\left(\operatorname{div}_{\Gamma}, \Gamma\right)=\left\{\mathbf{v} \in \mathbf{H}_{\times}^{-1 / 2}(\Gamma): \operatorname{div}_{\Gamma} \mathbf{v} \in\right.$ $\left.\mathrm{H}^{-1 / 2}(\Gamma)\right\}$ with $\mathbf{H}_{\times}^{-1 / 2}(\Gamma)$ is the dual space of the Hilbert space $\mathbf{H}_{\times}^{1 / 2}(\Gamma)=\gamma_{t}\left(\mathrm{H}^{1}(\Omega)\right)$ with respect to the pairing $\left\langle\gamma_{t} \mathbf{v}, \gamma_{t} \mathbf{u}\right\rangle_{\tau, \Gamma}=\int_{\Gamma}(\operatorname{curl} \mathbf{v} \cdot \mathbf{u}-\mathbf{v} \cdot \operatorname{curl} \mathbf{u}) \mathrm{d} x$.

Any electromagnetic field in $\Omega^{+}$which is a sum of a plane wave $\left(\mathbf{E}^{\text {inc }}, \mathbf{H}^{\text {inc }}\right)$ and of a radiating field $(\mathbf{E}, \mathbf{H})$ is uniquely determined by the knowledge of the two equivalent currents,

$$
\mathbf{J}(x)=\mathbf{n} \times \mathbf{H}_{\mid \Gamma}(x) \text { and } \mathbf{M}(x)=-\mathbf{n} \times \mathbf{E}_{\mid \Gamma}(x),
$$

through the well known Stratton-Chu formulae $[7,15,25]$

$$
\begin{cases}\mathbf{E}(x)=i Z_{0} \tilde{\mathrm{T}} \mathbf{J}(x)+\tilde{\mathrm{K}} \mathbf{M}(x) & \\ \mathbf{H}(x)=-\tilde{\mathrm{K}} \mathbf{J}(x)+i Z_{0}^{-1} \tilde{\mathrm{T}} \mathbf{M}(x) & x \in \Omega^{+},\end{cases}
$$

where the respective potentials $\tilde{T}$ and $\tilde{K}$ are defined by

$$
\begin{array}{rlrl}
\tilde{\mathrm{T}}: \mathbf{H}_{\times}^{-1 / 2}\left(\operatorname{div}_{\Gamma}, \Gamma\right) & \rightarrow & \mathbf{H}_{\mathrm{loc}}\left(\operatorname{curl}^{2}, \Omega^{+} \cup \Omega^{-}\right) \cap \mathbf{H}_{\mathrm{loc}}\left(\operatorname{div} 0, \Omega^{+} \cup \Omega^{-}\right) \\
\mathbf{J} & \mapsto \tilde{\mathrm{T}} \mathbf{J}(x)=k \int_{\Gamma} G(x, y) \mathbf{J}(y) \mathrm{d} \Gamma(y)+\frac{1}{k} \int_{\Gamma} \vec{\nabla}_{x} G(x, y) \operatorname{div}_{\Gamma} \mathbf{J}(y) \mathrm{d} \Gamma(y) \\
\tilde{\mathrm{K}}: \mathbf{H}_{\times}^{-1 / 2}\left(\operatorname{div}_{\Gamma}, \Gamma\right) & \rightarrow & \mathbf{H}_{\mathrm{loc}}\left(\operatorname{curl}^{2}, \Omega^{+} \cup \Omega^{-}\right) \cap \mathbf{H}_{\mathrm{loc}}\left(\operatorname{div} 0, \Omega^{+} \cup \Omega^{-}\right) \\
\mathbf{J}(x) & & \tilde{\mathrm{K}} \mathbf{J}(x)=\int_{\Gamma} \vec{\nabla}_{y} G(x, y) \times \mathbf{J}(y) \mathrm{d} \Gamma(y)
\end{array}
$$

and $G(x, y)$ is the fundamental solution for the radiating solution of the 3 -D Helmholtz equation

$$
G(x, y)=\frac{\exp (i k|x-y|)}{4 \pi|x-y|}
$$

The tangential traces on $\Gamma$ of the potentials $\tilde{\mathrm{T}}$ and $\tilde{\mathrm{K}}$ are known and one has $[7,25]$

$$
\begin{aligned}
& \mathbf{n} \times\left(\mathbf{E}_{\mid \Gamma} \times \mathbf{n}\right)(x)=i Z_{0} \mathrm{~T} \mathbf{J}(x)+\mathrm{KM}(x)+\frac{1}{2} \mathbf{n} \times \mathbf{M}(x), \\
& \mathbf{n} \times\left(\mathbf{H}_{\mid \Gamma} \times \mathbf{n}\right)(x)=-\mathrm{KJ}(x)-\frac{1}{2} \mathbf{n} \times \mathbf{J}(x)+i Z_{0}^{-1} \mathrm{TM}(x),
\end{aligned}
$$


where $\mathrm{T}$ and $\mathrm{K}$ are defined by

$$
\begin{aligned}
\mathrm{T}: \mathbf{H}_{\times}^{-1 / 2}\left(\operatorname{div}_{\Gamma}, \Gamma\right) & \rightarrow \mathbf{H}_{\times}^{-1 / 2}\left(\operatorname{curl}_{\Gamma}, \Gamma\right) \\
\mathbf{J} & \mapsto\left\{\gamma_{t}(\tilde{\mathrm{T}} \mathbf{J}) \times \mathbf{n}\right\}_{\Gamma} \\
\mathrm{K}: \mathbf{H}_{\times}^{-1 / 2}\left(\operatorname{div}_{\Gamma}, \Gamma\right) & \rightarrow \mathbf{H}_{\times}^{-1 / 2}\left(\operatorname{curl}_{\Gamma}, \Gamma\right) \\
\mathbf{J} & \mapsto\left\{\gamma_{t}(\tilde{\mathrm{K}} \mathbf{J}) \times \mathbf{n}\right\}_{\Gamma}
\end{aligned}
$$

where $\left\{\gamma_{t} A \times \mathbf{n}\right\}=\frac{1}{2}\left(\mathbf{n}^{+} \times\left(A \times \mathbf{n}^{+}\right)+\mathbf{n}^{-} \times\left(A \times \mathbf{n}^{-}\right)\right)$and $\mathbf{H}_{\times}^{-1 / 2}\left(\operatorname{curl}_{\Gamma}, \Gamma\right)=\left\{\mathbf{v} \in \mathbf{H}_{\perp}^{-1 / 2}(\Gamma): \operatorname{curl}_{\Gamma} \mathbf{v} \in \mathrm{H}^{-1 / 2}(\Gamma)\right\}$ is the dual space $\mathbf{H}_{\times}^{-1 / 2}\left(\operatorname{div}_{\Gamma}, \Gamma\right)$ with $\mathbf{H}_{\perp}^{-1 / 2}(\Gamma)$ is the dual space of the Hilbert space $\mathbf{H}_{\perp}^{1 / 2}(\Gamma)=\mathbf{n} \times \gamma_{t}\left(\mathrm{H}^{1}(\Omega)\right)$.

Using (2.5), we obtain

$$
\begin{aligned}
\mathbf{M} & =\frac{1}{2} \mathbf{M}-\mathbf{n} \times \mathrm{KM}-i Z_{0} \mathbf{n} \times \mathrm{T} \mathbf{J} \\
\mathbf{n} \times \mathbf{J} & =\frac{1}{2} \mathbf{n} \times \mathbf{J}+\mathrm{K} \mathbf{J}-i Z_{0}^{-1} \mathrm{TM}
\end{aligned}
$$

These two relations hold whatever the boundary condition on $\Gamma$ is. There are not independent: except for some exceptional values of $k$ (interior resonance), they are indeed equivalent. When impedance boundary condition is considered, we have to add the boundary condition (2.3) or equivalently

$$
\mathbf{n} \times \mathbf{M}(x)=\eta Z_{0} \mathbf{J}(x)+\mathbf{g}(x) \quad \text { for } x \in \Gamma .
$$

The two unknowns $\mathbf{J}, \mathbf{M}$ have to be determined using the four previous equations. Several boundary integral equations can be constructed to determine the currents, all of them amounts to combine (2.11) and (2.12) to get an equation with a unique solution. The derivations of some of these equations can be found for example in $[4]$.

\section{WELL-CONDITIONED INTEGRAL EQUATION}

\subsection{A general approach}

In this part, we present a general approach to construct an inherently well-conditioned integral equation. For that, we follow the reasoning which is used in $[1,17]$.

We consider the generic scattering problem: find the radiating electromagnetic field $(\mathbf{E}, \mathbf{H})$ in $\Omega^{+}$

- solution of the Maxwell equations (2.1) with radiation condition (2.2);

- and subjected to a boundary condition $\mathbf{B}(\mathbf{J}, \mathbf{M})=\mathbf{g}$ on the boundary $\Gamma$.

For example, the perfect conducting object corresponds to $\mathbf{B}(\mathbf{J}, \mathbf{M})=\mathbf{M}=-\mathbf{E}^{\mathrm{inc}} \times \mathbf{n}$ and the purely impedance case corresponds to $\mathbf{B}(\mathbf{J}, \mathbf{M})=\mathbf{M}+\eta Z_{0} \mathbf{n} \times \mathbf{J}=-\mathbf{n} \times \mathbf{g}=\tilde{\mathbf{g}}$.

Now, if we assume that our generic problem is well-posed, then one can formally define an operator $\mathbf{Y}^{e x}$ which links the data $\mathbf{g}$ to the electromagnetic currents $(\mathbf{J}, \mathbf{M})$ solution of the problem in this way:

$$
(\mathbf{J}, \mathbf{M})=\mathbf{Y}^{e x} \mathbf{g}=\left(\mathbf{Y}_{J}^{e x}, \mathbf{Y}_{M}^{e x}\right) \mathbf{g}
$$


Recall that the electromagnetic fields solution of the Maxwell equations (2.1) with radiation condition (2.2) can be parameterized by the currents $(\mathbf{J}, \mathbf{M})$ via (2.6) and so if we introduce (3.1) in these equations, we obtain:

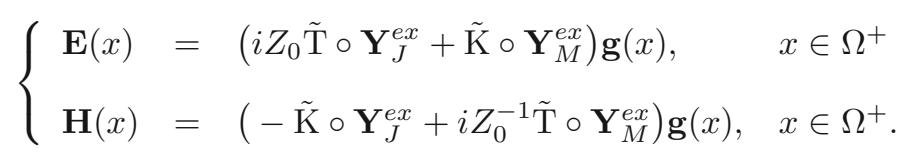

Finally, if we form the boundary condition by using (3.2), we obviously obtain:

$$
\mathbf{B} \circ \mathbf{P} \circ \mathbf{Y}^{e x} \mathbf{g}=\mathbf{g}
$$

where $\mathbf{P}$ is the Calderón projector defined by (2.11):

$$
\mathbf{P}=\left[\begin{array}{cc}
\frac{1}{2} \mathrm{Id}-\mathbf{n} \times \mathrm{K} & i Z_{0}^{-1} \mathbf{n} \times \mathrm{T} \\
-i Z_{0} \mathbf{n} \times \mathrm{T} & \frac{1}{2} \mathrm{Id}-\mathbf{n} \times \mathrm{K}
\end{array}\right]
$$

where Id is the identity operator.

So, we obtain the crucial relation:

$$
\mathbf{B} \circ \mathbf{P} \circ \mathbf{Y}^{e x}=\mathrm{Id} .
$$

In conclusion, one can parameterized the electromagnetic field by a density $\mathbf{u}$,

$$
\left\{\begin{array}{l}
\mathbf{E}=\left(i Z_{0} \tilde{\mathrm{T}} \circ \mathbf{Y}_{J}^{e x}+\tilde{\mathrm{K}} \circ \mathbf{Y}_{M}^{e x}\right) \mathbf{u} \\
\mathbf{H}=\left(-\tilde{\mathrm{K}} \circ \mathbf{Y}_{J}^{e x}+i Z_{0}^{-1} \tilde{\mathrm{T}} \circ \mathbf{Y}_{M}^{e x}\right) \mathbf{u}
\end{array}\right.
$$

which is the solution of the integral equation:

$$
\mathbf{B} \circ \mathbf{P} \circ \mathbf{Y}^{e x} \mathbf{u}=\mathbf{g}
$$

In this particular case, $\mathbf{u}=\mathbf{g}$ because $\mathbf{B} \circ \mathbf{P} \circ \mathbf{Y}^{e x}=\mathrm{Id}$.

Now, we are going to exploit the properties (3.4), (3.5) and (3.6) to formally construct well-conditioned integral equations. Since the operator $\mathbf{Y}^{e x}$ is generally unknown in practice, we assume that we possess an approximation $\mathbf{Y}$ of this operator. The idea is to substitute $\mathbf{Y}=\left(\mathbf{Y}_{J}, \mathbf{Y}_{M}\right)$ to $\mathbf{Y}^{e x}$ in (3.5) and (3.6). For that, we choose to parameterize the radiating electromagnetic field by a density $\mathbf{u}$,

$$
\left\{\begin{array}{l}
\mathbf{E}=\left(i Z_{0} \tilde{\mathrm{T}} \circ \mathbf{Y}_{J}+\tilde{\mathrm{K}} \circ \mathbf{Y}_{M}\right) \mathbf{u} \\
\mathbf{H}=\left(-\tilde{\mathrm{K}} \circ \mathbf{Y}_{J}+i Z_{0}^{-1} \tilde{\mathrm{T}} \circ \mathbf{Y}_{M}\right) \mathbf{u}
\end{array}\right.
$$

which is the solution of the integral equation:

$$
\mathbf{B} \circ \mathbf{P} \circ \mathbf{Y u}=\mathbf{g}
$$

Now, let us give some important remarks:

- The electromagnetic field defined by (3.7) is always a radiating field i.e. verifying the Maxwell equations and the radiation condition at the infinity. 
- For all operator $\mathbf{Y}$ such that the integral equation (3.8) is well-posed, the electromagnetic fields $(\mathbf{E}, \mathbf{H})$ defined by (3.7) and (3.8) is the exact field of our initial problem. It is true even if $\mathbf{Y}$ is a "bad" approximation of the optimal operator $\mathbf{Y}^{e x}$.

- If $\mathbf{Y}$ is "sufficiently close" on $\mathbf{Y}^{e x}$, one expects that the integral operator $\mathbf{B} \circ \mathbf{P} \circ \mathbf{Y}$ leads to a wellconditioned linear system after discretization.

- The currents defined by $\mathbf{J}_{u}=\mathbf{Y}_{J} \mathbf{u}$ and $\mathbf{M}_{u}=\mathbf{Y}_{M} \mathbf{u}$ are fictitious.

\subsection{Application to the impedance problem}

\subsubsection{Formal derivation}

In our problem, the boundary condition is:

$$
\mathbf{B}(\mathbf{J}, \mathbf{M})=\mathbf{M}+\eta Z_{0} \mathbf{n} \times \mathbf{J}=\mathbf{g} \text { on } \Gamma .
$$

The existence and the uniqueness (Thm. 2.1) of the solution of problem (2.1)-(2.2)-(2.3) induce the existence of the operator $\mathbf{Y}^{e x}=\left(\mathbf{Y}_{M}^{e x}, \mathbf{Y}_{J}^{e x}\right)$ and in particular, we have

(3.10) leads to a new expression of the operator $\mathbf{Y}^{\text {ex }}$

$$
\mathbf{M}+\eta Z_{0} \mathbf{n} \times \mathbf{J}=\left(\mathbf{Y}_{M}^{e x}+\eta Z_{0} \mathbf{n} \times \mathbf{Y}_{J}^{e x}\right) \mathbf{g}=\mathbf{g} \quad \text { on } \Gamma .
$$

$$
\mathbf{Y}^{e x}=\left(\mathrm{Id}-\eta Z_{0} \mathbf{n} \times \mathbf{Y}_{J}^{e x}, \mathbf{Y}_{J}^{e x}\right)
$$

Consequently, a candidate for the approximation operator $\mathbf{Y}$ can be chosen as:

$$
\mathbf{Y}=\left(\mathrm{Id}-\eta Z_{0} \mathbf{n} \times \mathbf{Y}_{J}, \mathbf{Y}_{J}\right)
$$

where $\mathbf{Y}_{J}$ is an approximation of $\mathbf{Y}_{J}^{e x}$.

Now, we want to define an approximation of $\mathbf{Y}_{J}^{e x}$. For that, we introduce the so-called exact exterior admittance or Stecklov-Poincaré operator $\mathscr{Y}^{e x}$ of the surface $\Gamma$. Recall that the operator is defined in this way: let $(\mathbf{u}, \mathbf{v})$ be the solution of the well-posed problem:

$$
\left\{\begin{array}{llll}
\operatorname{curl} \mathbf{u}-i k Z_{0} \mathbf{v} & = & 0 & \text { in } \Omega^{+} \\
\operatorname{curl} \mathbf{v}+i k Z_{0}^{-1} \mathbf{u} & = & 0 & \text { in } \Omega^{+} \\
\mathbf{n} \times \mathbf{u} \in \mathbf{H}_{\times}^{-1 / 2}\left(\operatorname{div}_{\Gamma}, \Gamma\right) \text { fixed on } \Gamma & & \\
+ \text { radiation condition at infinity. } & &
\end{array}\right.
$$

The operator $\mathscr{Y}^{e x}$ corresponds to the operator which from $\mathbf{n} \times \mathbf{v}$ gives $\mathbf{n} \times \mathbf{u}=-\mathscr{Y}^{e x}(\mathbf{n} \times \mathbf{v})$. In particular, the current $(\mathbf{M}, \mathbf{J})$ on $\Gamma$ induce by the field solution of the problem (2.1)-(2.3)-(2.2) are linked by the relation:

$$
\mathbf{M}=\mathscr{Y}^{e x}(\mathbf{J}) .
$$

By using (3.9) and (3.14), one obtains a new expression of $\mathbf{Y}_{J}^{e x}$ :

$$
\mathbf{Y}_{J}^{e x}=\left(\mathscr{Y}^{e x}+\eta Z_{0} \mathbf{n} \times \mathrm{Id}\right)^{-1} .
$$

In conclusion, if one possesses an approximation $\mathscr{Y}$ of the admittance, we choose as candidate for the approximation of $\mathbf{Y}^{e x}$ :

$$
\left\{\begin{aligned}
\mathbf{Y} & =\left(\mathrm{Id}-\eta Z_{0} \mathbf{n} \times \mathbf{Y}_{J}, \mathbf{Y}_{J}\right) \\
\mathbf{Y}_{J} & =\left(\mathscr{Y}+\eta Z_{0} \mathbf{n} \times \mathrm{Id}\right)^{-1}
\end{aligned}\right.
$$


and the integral equation (3.8) becomes: find $\mathbf{u} \in \mathbf{H}_{\times}^{-1 / 2}\left(\operatorname{div}_{\Gamma}, \Gamma\right)$ such that

$$
\mathscr{Z} \mathbf{u} \equiv \mathbf{B} \circ \mathbf{P} \circ \mathbf{Y u}=\left(\frac{1}{2} \mathrm{Id}-\mathbf{n} \times \mathrm{K} \circ \mathbf{Y}_{M}-i Z_{0} \mathbf{n} \times \mathrm{T} \circ \mathbf{Y}_{J}+\eta Z_{0} \mathrm{~K} \circ \mathbf{Y}_{J}-i \eta \mathrm{T} \circ \mathbf{Y}_{M}\right) \mathbf{u}=\mathbf{g}
$$

\subsubsection{An approximation of the admittance for a smooth surface}

We choose an approximation based on by a microlocal analysis of the problem (3.13). This kind of approach has been already used by Antoine and Darbas for the construction of analytical preconditioners in electromagnetism for perfectly conductor objects. It is based on the knowledge of principal symbol of the admittance of a smooth surface and on an efficient approximation of the square root operator found in this symbol. They obtain an approximate operator which act on all zones: hyperbolic, elliptic and creeping zones. It is a regularization of the square root by an adequate small damping parameter which allows to correctly take into account the creeping modes. We will give the results which we briefly need and we refer to [16] for more details.

The principal symbol $\sigma\left(\mathscr{Y}^{e x}\right)$ of $\mathscr{Y}^{e x}$ is [2]

$$
\sigma\left(\mathscr{Y}^{e x}\right)\left(\theta_{2}, \theta_{3}, k\right)=-Z_{0}\left(1-\frac{|\theta|^{2}}{k^{2}}\right)^{-1 / 2}\left(\begin{array}{cc}
-\frac{\theta_{2} \theta_{3}}{k^{2}} & 1-\frac{\theta_{3}^{2}}{k^{2}} \\
-1+\frac{\theta_{2}^{2}}{k^{2}} & \frac{\theta_{2} \theta_{3}}{k^{2}}
\end{array}\right)
$$

where $\left(\theta_{2}, \theta_{3}\right)$ is the dual variable of a particular parameterization of $\Gamma,|\theta|^{2}=\theta_{2}^{2}+\theta_{3}^{2}$ and $k$ is the dual variable of the time $t$.

Now, if one returns in the primal variables by a inverse Fourier transform in space, one obtains the following approximation of $\mathscr{Y}^{e x}$ :

$$
\begin{aligned}
\mathscr{Y}: \mathbf{H}^{-1 / 2}\left(\operatorname{div}_{\Gamma}, \Gamma\right) & \rightarrow \mathbf{H}^{-1 / 2}\left(\operatorname{div}_{\Gamma}, \Gamma\right) \\
\mathbf{v} & \mapsto \mathscr{Y} \mathbf{u}=-Z_{0}\left(\operatorname{Id}+\frac{\vec{\Delta}_{\Gamma}}{k^{2}}\right)^{-\frac{1}{2}}\left(\operatorname{Id}-\frac{1}{k^{2}} \operatorname{curl}_{\Gamma} \operatorname{curl}_{\Gamma}\right) \circ \mathbf{n} \times \mathbf{v}
\end{aligned}
$$

where $\mathbf{H}^{-1 / 2}\left(\operatorname{div}_{\Gamma}, \Gamma\right)=\left\{\mathbf{u} \in \mathbf{H}_{t}^{-1 / 2}: \operatorname{div}_{\Gamma} \mathbf{u} \in H^{-1 / 2}\right\}$ is the classical space for a smooth surface.

For our convenience, we define another expression of $\mathscr{Y}$. For that, we consider the Helmholtz decomposition of tangential fields belonging to $\mathbf{H}^{-1 / 2}\left(\operatorname{div}_{\Gamma}, \Gamma\right)$ : For each $\mathbf{v} \in \mathbf{H}^{-1 / 2}\left(\operatorname{div}_{\Gamma}, \Gamma\right)$, there exists two scalar potential $\psi_{v} \in H^{1 / 2}(\Gamma)$ and $\phi_{v} \in H^{3 / 2}(\Gamma)$ such that:

$$
\mathbf{v}=\mathbf{n} \times \nabla_{\Gamma} \psi_{v}+\nabla_{\Gamma} \phi_{v}
$$

and by reading $\mathscr{Y}$ as $2 \times 2$ matrix of operator and by posing $\mathbf{w}=\mathbf{n} \times \nabla_{\Gamma} \psi_{w}+\nabla_{\Gamma} \phi_{w}=\mathscr{Y} \mathbf{v}$, one can write:

$$
\left(\begin{array}{c}
\psi_{w} \\
\phi_{w}
\end{array}\right)=Z_{0}\left(\begin{array}{cc}
0 \\
-\left(1+\frac{\Delta_{\Gamma}}{k^{2}}\right)^{-\frac{1}{2}} & \left(1+\frac{\Delta_{\Gamma}}{k^{2}}\right)^{\frac{1}{2}} \\
0
\end{array}\right)\left(\begin{array}{c}
\psi_{v} \\
\phi_{v}
\end{array}\right)
$$

The operator $i k\left(1+\frac{\Delta_{\Gamma}}{k^{2}}\right)^{\frac{1}{2}}$ corresponds to the Dirichlet-To-Neumann operator for the acoustic scattering problem [3]. For an efficient approximation of this one, we introduce a damping parameter $\varepsilon$ in order to perturb the wavenumber $k$ by $k_{\varepsilon}=k+\mathrm{i} \varepsilon$ and so we regularize the singularity of the square root at the level 
of the transition region of glancing rays. A suitable value of $\varepsilon$ has been determined in [16]: $\varepsilon=0.4 k^{-1 / 3} \mathscr{H}^{-2 / 3}$ where $\mathscr{H}$ is the mean curvature of $\Gamma$. This value is optimum for the spherical geometries.

Finally, we explain how to compute accurately the square root. Recall that the square-root operator $S Q R=\left(1+\frac{\Delta_{\Gamma}}{k_{\varepsilon}^{2}}\right)^{\frac{1}{2}}$ is a non-local pseudo-differential operator. To realize an efficient estimation of $S Q R$, we use the technique based on a Padé expansion of the square root and a rotating branch-cut technique [23] ( $\theta$ corresponds to the angle of the rotation):

$$
\left(1+\frac{\Delta_{\Gamma}}{k_{\varepsilon}^{2}}\right)^{\frac{1}{2}} \approx A_{0}+\sum_{j=1}^{p} A_{j} \frac{\Delta_{\Gamma}}{k_{\varepsilon}^{2}}\left(I+B_{j} \frac{\Delta_{\Gamma}}{k_{\varepsilon}^{2}}\right)^{-1}
$$

where $A_{0}=\mathrm{e}^{\mathrm{i} \theta / 2} R_{p}\left(\mathrm{e}^{-\mathrm{i} \theta}-1\right), A_{j}=\mathrm{e}^{-\mathrm{i} \theta / 2} a_{j} /\left(1+b_{j}\left(\mathrm{e}^{-\mathrm{i} \theta}-1\right)\right)^{2}, B_{j}=\mathrm{e}^{-\mathrm{i} \theta / 2} b_{j} /\left(1+b_{j}\left(\mathrm{e}^{-\mathrm{i} \theta}-1\right)\right)$ with $R_{p}(z)=1+\sum_{j=1}^{p} a_{j} z /\left(1+b_{j} z\right), a_{j}=2 /(2 p+1) \sin ^{2}(j \pi /(2 p+1))$ and $b_{j}=\cos ^{2}(j \pi /(2 p+1))$.

\subsubsection{More tractable approximations of $\mathscr{Y}_{\mathrm{J}}$ and $\mathscr{Y} \mathrm{M}$ on a smooth surface}

By pursuing our work with the Helmholtz potentials, we are going to give a new approximation $\mathbf{Y}$. For that, we look at the action of $\mathbf{Y}$ (3.16) on tangential vector field $\mathbf{u} \in \mathbf{H}_{T}^{0}(\Gamma)$ by using the Helmholtz decomposition.

First, if one poses $\mathbf{J}_{u}=\mathbf{Y}_{J} \mathbf{u} \in \mathbf{H}^{-1 / 2}\left(\operatorname{div}_{\Gamma}, \Gamma\right) \cap \mathbf{H}_{T}^{0}(\Gamma)$ and $\mathbf{M}_{u}=\mathbf{Y}_{M} \mathbf{u} \in \mathbf{H}^{-1 / 2}\left(\operatorname{div}_{\Gamma}, \Gamma\right) \cap \mathbf{H}_{T}^{0}(\Gamma)$ (these regularities are suggested by Thm. 2.1), it is easy to see that (3.16) can be write in this way:

$$
\left\{\begin{array}{l}
\mathbf{u}=\mathbf{M}_{u}+Z_{0} \eta \mathbf{n} \times \mathbf{J}_{u} \\
\mathbf{M}_{u}=\mathscr{Y} \mathbf{J}_{u} .
\end{array}\right.
$$

Secondly, by using the Helmholtz decompositions:

$$
\left\{\begin{array}{lll}
\mathbf{u} \in \mathbf{H}_{T}^{0}(\Gamma) & \mapsto \mathbf{u}=\mathbf{n} \times \nabla_{\Gamma} \psi_{u}+\nabla_{\Gamma} \phi_{u} & \text { with } \psi_{u}, \phi_{u} \in H^{1}(\Gamma) \\
\mathbf{M}_{u} \in \mathbf{H}^{-1 / 2}\left(\operatorname{div}_{\Gamma}, \Gamma\right) \cap \mathbf{H}_{T}^{0}(\Gamma) & \mapsto \mathbf{M}_{u}=\mathbf{n} \times \nabla_{\Gamma} \psi_{\mathbf{M}_{u}}+\nabla_{\Gamma} \phi_{\mathbf{M}_{u}} & \text { with }\left(\psi_{\mathbf{M}_{u}}, \phi_{\mathbf{M}_{u}}\right) \in H^{1}(\Gamma) \times H^{3 / 2}(\Gamma) \\
\mathbf{J}_{u} \in \mathbf{H}^{-1 / 2}\left(\operatorname{div}_{\Gamma}, \Gamma\right) \cap \mathbf{H}_{T}^{0}(\Gamma) & \mapsto \mathbf{J}_{u}=\mathbf{n} \times \nabla_{\Gamma} \psi_{\mathbf{J}_{u}}+\nabla_{\Gamma} \phi_{\mathbf{J}_{u}} & \text { with }\left(\psi_{\mathbf{J}_{u}}, \phi_{\mathbf{J}_{u}}\right) \in H^{1}(\Gamma) \times H^{3 / 2}(\Gamma)
\end{array}\right.
$$

the first equation of (3.23) leads to

$$
\mathbf{n} \times \nabla_{\Gamma} \psi_{\mathbf{u}}+\nabla_{\Gamma} \phi_{\mathbf{u}}=\mathbf{n} \times \nabla_{\Gamma} \psi_{\mathbf{M}_{u}}+\nabla_{\Gamma} \phi_{\mathbf{M}_{u}}+Z_{0} \eta\left(-\nabla_{\Gamma} \psi_{\mathbf{J}_{u}}+\mathbf{n} \times \nabla_{\Gamma} \phi_{\mathbf{J}_{u}}\right)
$$

and by formally applying $\operatorname{curl}_{\Gamma}$ and $\operatorname{div}_{\Gamma}$ to $(3.25)$, we obtain:

$$
\begin{aligned}
& \psi_{\mathbf{u}}=\psi_{\mathbf{M}_{u}}+Z_{0} \Delta_{\Gamma}^{-1}\left(\operatorname{curl}_{\Gamma}\left(\eta \nabla_{\Gamma} \psi_{\mathbf{J}_{u}}\right)\right)-Z_{0} \Delta_{\Gamma}^{-1}\left(\operatorname{curl}_{\Gamma}\left(\eta \mathbf{n} \times \nabla_{\Gamma} \phi_{\mathbf{J}_{u}}\right)\right) \\
& \phi_{\mathbf{u}}=\phi_{\mathbf{M}_{u}}-Z_{0} \Delta_{\Gamma}^{-1}\left(\operatorname{div}_{\Gamma}\left(\eta \nabla_{\Gamma} \psi_{\mathbf{J}_{u}}\right)\right)+Z_{0} \Delta_{\Gamma}^{-1}\left(\operatorname{div}_{\Gamma}\left(\eta \mathbf{n} \times \nabla_{\Gamma} \phi_{\mathbf{J}_{u}}\right)\right)
\end{aligned}
$$

where $\Delta_{\Gamma}^{-1}$ is the Moore-Penrose pseudo-inverse of the Laplace-Beltrami operator.

Now by using the second equation of (3.23) as well as (3.21), we get the system of two equations:

$$
\left\{\begin{aligned}
\frac{\psi_{\mathbf{u}}}{Z_{0}} & =\left(1+\frac{\Delta_{\Gamma}}{k_{\varepsilon}^{2}}\right)^{\frac{1}{2}} \phi_{\mathbf{J}_{u}}+\Delta_{\Gamma}^{-1}\left(\operatorname{rot}_{\Gamma}\left(\eta \nabla_{\Gamma} \psi_{\mathbf{J}_{u}}\right)\right)-\Delta_{\Gamma}^{-1}\left(\operatorname{rot}_{\Gamma}\left(\eta \mathbf{n} \times \nabla_{\Gamma} \phi_{\mathbf{J}_{u}}\right)\right) \\
-\frac{\phi_{\mathbf{u}}}{Z_{0}} & =\left(1+\frac{\Delta_{\Gamma}}{k_{\varepsilon}^{2}}\right)^{-\frac{1}{2}} \psi_{\mathbf{J}_{u}}+\Delta_{\Gamma}^{-1}\left(\operatorname{div}_{\Gamma}\left(\eta \nabla_{\Gamma} \psi_{\mathbf{J}_{u}}\right)\right)-\Delta_{\Gamma}^{-1}\left(\operatorname{div}_{\Gamma}\left(\eta \mathbf{n} \times \nabla_{\Gamma} \phi_{\mathbf{J}_{u}}\right)\right) .
\end{aligned}\right.
$$


We remark that if the impedance function $\eta$ is constant, then these two equations are decoupled in the sense that the first equation only depends on $\phi_{\mathbf{J}_{u}}$ and the second one on $\psi_{\mathbf{J}_{u}}$ since $\operatorname{rot}_{\Gamma}\left(\eta \nabla_{\Gamma} \psi_{\mathbf{J}_{u}}\right)=\operatorname{div}_{\Gamma}(\eta \mathbf{n} \times$ $\left.\nabla_{\Gamma} \phi_{\mathbf{J}_{u}}\right)=0$. So, by remembering of the second important remark made at the end of the section entitled "A general approach" and since $\eta$ is generally chosen to be piecewise constant, we consider a new "less complicated" approximation $\mathbf{Y}_{J}$ which is defined by:

$$
\left\{\begin{aligned}
\frac{\psi_{\mathbf{u}}}{Z_{0}} & =\left(1+\frac{\Delta_{\Gamma}}{k_{\varepsilon}^{2}}\right)^{\frac{1}{2}} \phi_{\mathbf{J}_{u}}-\Delta_{\Gamma}^{-1}\left(\operatorname{rot}_{\Gamma}\left(\eta \mathbf{n} \times \nabla_{\Gamma} \phi_{\mathbf{J}_{u}}\right)\right) \\
-\frac{\phi_{\mathbf{u}}}{Z_{0}} & =\left(1+\frac{\Delta_{\Gamma}}{k_{\varepsilon}^{2}}\right)^{-\frac{1}{2}} \psi_{\mathbf{J}_{u}}+\Delta_{\Gamma}^{-1}\left(\operatorname{div}_{\Gamma}\left(\eta \nabla_{\Gamma} \psi_{\mathbf{J}_{u}}\right)\right) .
\end{aligned}\right.
$$

Finally, we obtain $\mathbf{M}_{u}$ (or $\mathbf{Y}_{M}$ ) by taking $\mathbf{M}_{u}=\mathbf{u}-Z_{0} \eta \mathbf{n} \times \mathbf{J}_{u}=\mathbf{u}-Z_{0} \eta \mathbf{n} \times \mathbf{Y}_{\mathbf{J}} \mathbf{u}$.

Remark 3.1. The approximation (3.28) does not change the initial problem. Indeed, the representation formulae (2.6) always imply that $(\mathbf{E}, \mathbf{H})$ is solution of the problem (2.1)-(2.2) and the trace formulas derived of (2.11), the integral equation (3.17) and the operator $\mathbf{Y}_{M}$ give the boundary condition (2.3). The operator $\mathbf{Y}_{J}$ and consequently $\mathbf{Y}_{M}$ must be sufficiently close on the exact one in order to obtain an integral operator $\mathscr{Z}$ (defined in (3.17)) close to the identity operator. Numerically, these operators will particularly have an influence on the rate of convergence of the iterative solver.

\subsubsection{Case of a non-smooth surface}

At the begin of this paper, we have assumed that the domain $\Omega$ is a Lipschitz polyhedron which is simply connected and connected. Moreover, the integral framework and Theorem 2.1 has been given for this regularity assumption. Unfortunately, we are unable to currently determine an approximation $\mathbf{Y}$ of the optimal operator $\mathbf{Y}^{e x}$ in this context and that is why we have derived it for the smooth surface. Nevertheless, as we have already it said in many place, for all operator $\mathbf{Y},(3.7)$ and (3.8) define always the solution of the initial problem (2.1)-(2.2)-(2.3). Consequently, it is not absurd to consider the approximation defined in the smooth case for the non-smooth situation. It should all the same be made sure that (3.28) can be defined for the Lipschitz polyhedron. This does not pose any problem since Buffa et al. have proved that the space $\mathbf{H}_{\times}^{-1 / 2}\left(\operatorname{div}_{\Gamma}, \Gamma\right)$ admits the following Hodge decomposition [7]:

$$
\mathbf{H}_{\times}^{-1 / 2}\left(\operatorname{div}_{\Gamma}, \Gamma\right)=\mathbf{n} \times \nabla_{\Gamma}\left(H^{1 / 2}(\Gamma) / \mathbb{R}\right) \oplus \nabla_{\Gamma} \mathscr{H}(\Gamma)
$$

where $\mathscr{H}(\Gamma)=\left\{v \in H^{1}(\Gamma) / \mathbb{R}: \Delta_{\Gamma} v \in H^{-1 / 2}(\Gamma)\right\}$.

Remark 3.2. An Hodge decomposition of the space $\mathbf{H}_{\times}^{-1 / 2}\left(\operatorname{div}_{\Gamma}, \Gamma\right)$ can be also obtained in the multi-connected case [6].

Finally, an open question is the well-posedness of the integral equation (3.8) for the non-smooth surface. It is the same problem for the very popular Combined Field Integral Equation (CFIE) but this does not prevent its successful use in industrial contexts.

\section{WELL-POSEDNESS FOR SMOOTH SURFACE AND IMPEDANCE OPERATOR}

We assume that the surface $\Gamma$ is smooth and connected and that $\eta$ is constant in order to use the pseudodifferential calculus. The results can be extended to the case of a non-constant smooth impedance in a straightforward way.

In this section, we first prove that our choice leads to an equation which can be written under the form: $a^{*}$ identity + one compact operator where $a$ is closed on 1 i.e. $a^{*}$ identity $\approx$ identity. It is well known that this type of equation is well-adapted to an iterative resolution and that if the spectral behavior of the equation is well restored to the discrete level, then the convergence rate is independent to space and frequency refinement $[9,10]$. 
This result has already been proved in [18] for the metallic case i.e. $\eta=0$ and by using the same kind of techniques, we easily prove this property for $\eta \neq 0$. Then, we will prove the integral operator (3.17) is one-toone and consequently, a Fredholm alternative will allow us to assert the well-posed nature of the formulation.

As $\eta$ is assumed to be constant, (3.28) gives the simplified system:

$$
\left\{\begin{array}{l}
\psi_{\mathbf{J}_{u}}=-\frac{1}{Z_{0}}\left(\eta+\left(1+\frac{\Delta_{\Gamma}}{k_{\epsilon}^{2}}\right)^{-\frac{1}{2}}\right)^{-1} \phi_{\mathbf{u}}=Y_{\mathbf{J}}^{1} \psi_{\mathbf{u}} \\
\phi_{\mathbf{J}_{u}}=\frac{1}{Z_{0}}\left(\eta+\left(1+\frac{\Delta_{\Gamma}}{k_{\epsilon}^{2}}\right)^{\frac{1}{2}}\right)^{-1} \psi_{\mathbf{u}}=Y_{\mathbf{J}}^{2} \psi_{\mathbf{u}}
\end{array}\right.
$$

and

$$
\left\{\begin{aligned}
\psi_{\mathbf{M}_{u}} & =\left(1+\eta\left(1+\frac{\Delta_{\Gamma}}{k_{\varepsilon}^{2}}\right)^{-\frac{1}{2}}\right)^{-1} \psi_{\mathbf{u}}=Y_{\mathbf{M}}^{1} \psi_{\mathbf{u}} \\
\phi_{\mathbf{M}_{u}} & =\left(1+\eta\left(1+\frac{\Delta_{\Gamma}}{k_{\varepsilon}^{2}}\right)^{\frac{1}{2}}\right)^{-1} \phi_{\mathbf{u}}=Y_{\mathbf{M}}^{2} \phi_{\mathbf{u}}
\end{aligned}\right.
$$

Proposition 4.1. We have

$$
\begin{aligned}
Y_{\mathbf{M}}^{1} & \sim \operatorname{Id} \bmod \Psi^{-1}(\Gamma) & Y_{\mathbf{J}}^{1} & \sim-\frac{1}{Z_{0} \eta} \operatorname{Id} \bmod \Psi^{-1}(\Gamma) \\
Y_{\mathbf{M}}^{2} & \sim \operatorname{op}\left(\frac{k_{\varepsilon}}{i \eta|\theta|}\right) \bmod \Psi^{-2}(\Gamma) & Y_{\mathbf{J}}^{2} & \sim \operatorname{op}\left(\frac{k_{\varepsilon}}{Z_{0} i|\theta|}\right) \bmod \Psi^{-2}(\Gamma)
\end{aligned}
$$

where $\Psi^{s}(\Gamma)$ denotes the class of pseudo-differential operators of order s defined on the closed surface $\Gamma, \operatorname{op}(\sigma)$ defines the pseudo-differential operator whose the symbol is $\sigma$ and $A \sim B \bmod \Psi^{-m}(\Gamma)$ with $m \in \mathbb{Z}$ means $\exists C \in \Psi^{-m}(\Gamma)$ such that $A-(B+C) \in \Psi^{-\infty}(\Gamma)$.

Proof. All these results are obtained in the same way. We only detail the calculus for $Y_{\mathbf{M}}^{2}$ :

$$
\begin{aligned}
\sigma\left(Y_{\mathbf{M}}^{2}\right) & =\frac{1}{1+\eta \sqrt{1-\frac{|\theta|^{2}}{k_{\varepsilon}^{2}}}}=\frac{1}{1+i \eta \frac{|\theta|}{k_{\varepsilon}} \sqrt{1-\frac{k_{\varepsilon}^{2}}{|\theta|^{2}}}}=\frac{1}{1+i \eta \frac{|\theta|}{k_{\varepsilon}}\left(1+\sum_{j=1}^{+\infty} \frac{\lambda_{j}}{|\theta|^{2 j}}\right)} \\
& =\frac{1}{i \eta \frac{|\theta|}{k_{\varepsilon}}\left(\frac{k_{\varepsilon}}{i \eta|\theta|}+1+\sum_{j=1}^{+\infty} \frac{\lambda_{j}}{|\theta|^{2 j}}\right)}=\frac{k_{\varepsilon}}{i \eta|\theta|}\left(1+\sum_{j=1}^{+\infty}(-1)^{j} X^{j}\right)
\end{aligned}
$$

where $X=\frac{k_{\varepsilon}}{i \eta|\theta|}+\sum_{j=1}^{+\infty} \frac{\lambda_{j}}{|\theta|^{2 j}}$

This expansion is valid in the elliptic zone and for $|\theta| \rightarrow+\infty$.

Proposition 4.2. We have

$$
\begin{aligned}
& \mathbf{n} \times T \mathscr{Y}_{\mathbf{J}} \sim \frac{k_{\varepsilon}}{2 i Z_{0} k} \mathbf{n} \times \nabla_{\Gamma} \Delta_{\Gamma}^{-1} \operatorname{curl}_{\Gamma} \bmod \Psi^{-1}(\Gamma) \\
& T \mathscr{Y}_{\mathbf{M}} \sim-\frac{k_{\varepsilon}}{2 i \eta k} \nabla_{\Gamma} \Delta_{\Gamma}^{-1} \operatorname{div}_{\Gamma} \bmod \Psi^{-1}(\Gamma) .
\end{aligned}
$$


Proof. We only prove the first result. The second is obtained in the same way. First, we write $T$ under this straightforward form $T=k G+\frac{1}{k} \nabla_{\Gamma} G_{s} \operatorname{div}_{\Gamma}$ and $\mathbf{J}_{u}, \mathbf{M}_{u}$ in this way

$$
\begin{aligned}
& \mathbf{J}_{u}=\left(\mathbf{n} \times \nabla_{\Gamma} Y_{\mathbf{J}}^{1} \Delta_{\Gamma}^{-1} \operatorname{div}_{\Gamma}-\nabla_{\Gamma} Y_{\mathbf{J}}^{2} \Delta_{\Gamma}^{-1} \operatorname{curl}_{\Gamma}\right) \mathbf{u} \\
& \mathbf{M}_{u}=\left(-\mathbf{n} \times \nabla_{\Gamma} Y_{\mathbf{M}}^{1} \Delta_{\Gamma}^{-1} \operatorname{curl}_{\Gamma}+\nabla_{\Gamma} Y_{\mathbf{M}}^{2} \Delta_{\Gamma}^{-1} \operatorname{div}_{\Gamma}\right) \mathbf{u} .
\end{aligned}
$$

We then get:

$$
\begin{aligned}
\mathbf{n} \times T \mathbf{J}_{u}= & \left(k \mathbf{n} \times G+\frac{1}{k} \mathbf{n} \times \nabla_{\Gamma} G_{s} \operatorname{div}_{\Gamma}\right)\left(\mathbf{n} \times \nabla_{\Gamma} Y_{\mathbf{J}}^{1} \Delta_{\Gamma}^{-1} \operatorname{div}_{\Gamma}-\nabla_{\Gamma} Y_{\mathbf{J}}^{2} \Delta_{\Gamma}^{-1} \operatorname{curl}_{\Gamma}\right) \mathbf{u} \\
= & k \mathbf{n} \times G \mathbf{n} \times \nabla_{\Gamma} Y_{\mathbf{J}}^{1} \Delta_{\Gamma}^{-1} \operatorname{div}_{\Gamma} \mathbf{u} \\
& -k \mathbf{n} \times G \nabla_{\Gamma} Y_{\mathbf{J}}^{2} \Delta_{\Gamma}^{-1} \operatorname{curl}_{\Gamma} \mathbf{u}+\frac{1}{k} \mathbf{n} \times \nabla_{\Gamma} G_{s} \Delta_{\Gamma} Y_{\mathbf{J}}^{2} \Delta_{\Gamma}^{-1} \operatorname{curl}_{\Gamma} \mathbf{u} .
\end{aligned}
$$

Finally, by using these classical properties: $G_{s} \sim-\frac{1}{2|\theta|} \bmod \Psi^{-2}(\Gamma), G \sim-\frac{1}{2|\theta|} \operatorname{Id} \bmod \Psi^{-2}(\Gamma)$ and $\Delta_{\Gamma}^{-1} \sim$ $-4 G_{s}^{2} \bmod \Psi^{-3}(\Gamma)$, we immediately obtain:

$$
\begin{aligned}
\mathbf{n} \times T \mathscr{Y}_{\mathbf{J}} & \sim \frac{1}{k} \mathbf{n} \times \nabla_{\Gamma} G_{s} \Delta_{\Gamma} Y_{\mathbf{J}}^{2} \Delta_{\Gamma}^{-1} \operatorname{curl}_{\Gamma} \bmod \Psi^{-1}(\Gamma) \\
& \sim-\frac{2 k_{\varepsilon}}{i Z_{0} k} \mathbf{n} \times \nabla_{\Gamma} G_{s} \Delta_{\Gamma} G_{s} \Delta_{\Gamma}^{-1} \operatorname{curl}_{\Gamma} \bmod \Psi^{-1}(\Gamma) \\
& \sim-\frac{2 k_{\varepsilon}}{i Z_{0} k} \mathbf{n} \times \nabla_{\Gamma} G_{s}^{2} \operatorname{curl}_{\Gamma} \bmod \Psi^{-1}(\Gamma) \\
& \sim \frac{k_{\varepsilon}}{2 i Z_{0} k} \mathbf{n} \times \nabla_{\Gamma} \Delta_{\Gamma}^{-1} \operatorname{curl}_{\Gamma} \bmod \Psi^{-1}(\Gamma)
\end{aligned}
$$

Introducing (4.4) in (3.17) and as $K$ is pseudo-differential operator of order -3 [27], we obtain:

$$
\begin{aligned}
\mathscr{Z} & \sim \frac{1}{2} \mathrm{Id}-\frac{k_{\varepsilon}}{2 k} \mathbf{n} \times \nabla_{\Gamma} \Delta_{\Gamma}^{-1} \operatorname{curl}_{\Gamma}+\frac{k_{\varepsilon}}{2 k} \nabla_{\Gamma} \Delta_{\Gamma}^{-1} \operatorname{div}_{\Gamma} \bmod \Psi^{-1}(\Gamma) \\
& \sim\left(\frac{1}{2}+\frac{k_{\varepsilon}}{2 k}\right) \operatorname{Id} \bmod \Psi^{-1}(\Gamma)
\end{aligned}
$$

where $a=\frac{1}{2}+\frac{k_{\varepsilon}}{2 k}=1+\frac{\varepsilon}{2 k}$.

We still have to prove the injectivity of the operator in order to use a Fredholm alternative. For that, we again follow [18] by using the spectral decomposition of Laplace-Beltrami operator $\Delta_{\Gamma}$ on $\Gamma$ : let $\left(Y_{i}\right)_{i}$ be a basis of eigenvectors of $\Delta_{\Gamma}$, then we have $-\Delta_{\Gamma} Y_{i}=\lambda_{i} Y_{i}$ with $\lambda_{i} \geq 0$. Moreover $\left(Y_{i}\right)_{i}$ and $\left(\frac{\nabla_{\Gamma} Y_{i}}{\sqrt{\lambda_{i}}}, \frac{\mathbf{n} \times \nabla_{\Gamma} Y_{i}}{\sqrt{\lambda_{i}}}\right)_{i \geq 1}$ are orthogonal Hilbertian basis of $L^{2}(\Gamma)$ and $L_{T}^{2}(\Gamma)$ respectively.

Proposition 4.3. If the operator $\mathbf{Y}=\left(\mathbf{Y}_{M}, \mathbf{Y}_{J}\right)$ verifies the condition

$$
\Re\left(\int_{\Gamma} \mathbf{n} \times \mathbf{Y}_{J} \mathbf{u} \cdot \overline{\mathbf{Y}_{M} \mathbf{u}} \mathrm{d} \Gamma\right)>0 \text { for all } \mathbf{u} \in \mathbf{H}_{T}^{0}(\Gamma) \text { and } \mathbf{u} \neq 0
$$

then the equation (3.17) is one-to-one. 
Proof. First, if one takes a zero incident field, then one obviously has $\mathbf{E}_{\mid \Omega^{+}}=\mathbf{H}_{\mid \Omega^{+}}=0$. Since $\mathbf{n} \times\left(\mathbf{E}_{\Gamma}^{-} \times \mathbf{n}\right)=$ $-1 / 2 \mathbf{n} \times \mathbf{Y}_{M} \mathbf{u}+i Z_{0} T \mathbf{Y}_{J} \mathbf{u}+K \mathbf{Y}_{M} \mathbf{u}$ (- denotes the interior trace), we immediately get $\left(\mathbf{E}_{\Gamma}^{-} \times \mathbf{n}\right)=-\mathbf{Y}_{M} \mathbf{u}$. In the same way, we have $\mathbf{n} \times\left(\mathbf{H}_{\Gamma}^{-} \times \mathbf{n}\right)=1 /\left(i k Z_{0}\right)\left(\mathbf{n} \times\left(\operatorname{rot}\left(\mathbf{E}^{-}\right)_{\Gamma} \times \mathbf{n}\right)\right)=\mathbf{n} \times \mathbf{Y}_{J} \mathbf{u}$.

By using the Green formula on $\Omega^{-}$, we can derive $\left\|\operatorname{rot} \mathbf{E}^{-}\right\|_{0, \Omega^{-}}^{2}-k^{2}\left\|\mathbf{E}^{-}\right\|_{0, \Omega^{-}}^{2}=\int_{\Gamma}\left(\mathbf{n} \times\left(\operatorname{rot}\left(\mathbf{E}^{-}\right)_{\Gamma} \times \mathbf{n}\right)\right)$. $\overline{\left(\mathbf{E}^{-} \times \mathbf{n}\right)} \mathrm{d} \Gamma=-\left(i k Z_{0}\right) \int_{\Gamma}\left(\mathbf{n} \times \mathbf{Y}_{J} \mathbf{u}\right) \cdot \overline{\mathbf{Y}}_{M} \mathbf{u} \mathrm{d} \Gamma$. So, since $k$ is a real number, we obtain $\Re\left(\int_{\Gamma}\left(\mathbf{n} \times \mathscr{Y}_{\mathbf{J}} \mathbf{u}\right)\right.$. $\left.\overline{\mathscr{Y}_{\mathbf{M}} \mathbf{u}} \mathrm{d} \Gamma\right)=0$ and $(4.6)$ leads to $\mathbf{u}=0$.

Now, we prove (4.6) for our choice of operator $\mathbf{Y}$.

Proposition 4.4. If one chooses the operator $\mathbf{Y}$ defined in Section 3.2.3, then the condition

$$
\Re\left(\int_{\Gamma} \mathbf{n} \times \mathbf{Y}_{J} \mathbf{u} \cdot \overline{\mathbf{Y}_{M} \mathbf{u}} \mathrm{d} \Gamma\right)>0 \text { for all } \mathbf{u} \in \mathbf{H}_{T}^{0}(\Gamma) \text { and } \mathbf{u} \neq 0
$$

holds.

Proof. Let $\mathbf{u} \in \mathbf{H}_{T}^{0}(\Gamma)$ such that $\mathbf{u} \neq 0$. One can decompose $\mathbf{u}$ in this way: $\mathbf{u}=\sum_{i=1}^{\infty}\left(\alpha_{i} \frac{\mathbf{n} \times \nabla_{\Gamma} Y_{i}}{\sqrt{\lambda_{i}}}+\beta_{i} \frac{\nabla_{\Gamma} Y_{i}}{\sqrt{\lambda_{i}}}\right)$ where $\alpha_{i}, \beta_{i}$ are complex numbers. In particular, one can take $\psi_{\mathbf{u}}=\sum_{i=1}^{\infty} \alpha_{i} \frac{Y_{i}}{\sqrt{\lambda_{i}}}$ and $\phi_{\mathbf{u}}=\sum_{i=1}^{\infty} \beta_{i} \frac{Y_{i}}{\sqrt{\lambda_{i}}}$. (4.1) and (4.2) give us:

$$
\begin{aligned}
\psi_{\mathbf{J}_{u}} & =-\frac{1}{Z_{0}} \sum_{i=1}^{\infty} \beta_{i}\left(\eta+\left(1-\frac{\lambda_{i}}{k_{\varepsilon}^{2}}\right)^{-\frac{1}{2}}\right)^{-1} \frac{Y_{i}}{\sqrt{\lambda_{i}}}=-\frac{1}{Z_{0}} \sum_{i=1}^{\infty} \beta_{i}\left(\psi_{\mathbf{J}_{u}}\right)_{i} \frac{Y_{i}}{\sqrt{\lambda_{i}}} \\
\phi_{\mathbf{J}_{u}} & =\frac{1}{Z_{0}} \sum_{i=1}^{\infty} \alpha_{i}\left(\eta+\left(1-\frac{\lambda_{i}}{k_{\varepsilon}^{2}}\right)^{\frac{1}{2}}\right)^{-1} \frac{Y_{i}}{\sqrt{\lambda_{i}}}=\frac{1}{Z_{0}} \sum_{i=1}^{\infty} \alpha_{i}\left(\phi_{\mathbf{J}_{u}}\right)_{i} \frac{Y_{i}}{\sqrt{\lambda_{i}}} \\
\psi_{\mathbf{M}_{u}} & =\sum_{i=1}^{\infty} \alpha_{i}\left(1+\eta\left(1-\frac{\lambda_{i}}{k_{\varepsilon}^{2}}\right)^{-\frac{1}{2}}\right)^{-1} \frac{Y_{i}}{\sqrt{\lambda_{i}}}=\sum_{i=1}^{\infty} \alpha_{i}\left(\psi_{\mathbf{M}_{u}}\right)_{i} \frac{Y_{i}}{\sqrt{\lambda_{i}}} \\
\phi_{\mathbf{M}_{u}} & =\sum_{i=1}^{\infty} \beta_{i}\left(1+\eta\left(1-\frac{\lambda_{i}}{k_{\varepsilon}^{2}}\right)^{\frac{1}{2}}\right)^{-1} \frac{Y_{i}}{\sqrt{\lambda_{i}}}=\sum_{i=1}^{\infty} \beta_{i}\left(\phi_{\mathbf{M}_{u}}\right)_{i} \frac{Y_{i}}{\sqrt{\lambda_{i}}}
\end{aligned}
$$

(4.8) leads to $\int_{\Gamma}\left(\mathbf{n} \times \mathbf{Y}_{J} \mathbf{u}\right) \cdot \mathbf{Y}_{M} \mathbf{u} \mathrm{d} \Gamma=1 / Z_{0} \sum_{i=1}^{\infty}\left(\left|\beta_{i}\right|^{2}\left(\psi_{\mathbf{J}_{u}}\right)_{i} \overline{\left(\phi_{\mathbf{M}_{u}}\right)_{i}}+\left|\alpha_{i}\right|^{2}\left(\phi_{\mathbf{J}_{u}}\right)_{i} \overline{\left(\psi_{\mathbf{M}_{u}}\right)_{i}}\right)$. Since $\left(\psi_{\left.\mathbf{J}_{u}\right)_{i}} \overline{\left(\phi_{\left.\mathbf{M}_{u}\right)_{i}}\right.}=\right.$ $\left(1-\frac{\lambda_{i}}{k_{\varepsilon}^{2}}\right)^{\frac{1}{2}}\left|\left(1+\eta\left(1-\frac{\lambda_{i}}{k_{\varepsilon}^{2}}\right)^{\frac{1}{2}}\right)\right|^{-2},\left(\phi_{\mathbf{J}_{u}}\right)_{i} \overline{\left(\psi_{\left.\mathbf{M}_{u}\right)_{i}}\right.}=\left(1-\frac{\lambda_{i}}{k_{\varepsilon}^{2}}\right)^{-\frac{1}{2}}\left|\left(1+\eta\left(1-\frac{\lambda_{i}}{k_{\varepsilon}^{2}}\right)^{-\frac{1}{2}}\right)\right|^{-2}, \eta>0$ (see assumption Thm. 2.1) and $\Re\left(1-\frac{\lambda_{i}}{k_{\varepsilon}^{2}}\right)^{ \pm \frac{1}{2}}>0$ (for $k>0$ and $\varepsilon>0$ ), we finally get the result.

Finally, we have:

Theorem 4.5. If one chooses the operator $\mathbf{Y}$ defined in Section 3.2.3, then the integral equation (3.17) is well-posed for any frequency.

Proof. We use a basic Fredholm argument. 


\section{Discretization And Resolution}

After discretization, we want to iteratively solve (3.17). For that, we have to be able to form the matrixvector products defined by the operator $\mathscr{Z}$ and required by the iterative solver. But, the choice made for $\mathbf{Y}_{J}$ and $\mathbf{Y}_{M}$ does not allow us to directly discretize the operators $\mathrm{T} \circ \mathbf{Y}_{O P}$ and $\mathrm{K} \circ \mathbf{Y}_{O P}$ where $O P=\mathbf{Y}_{J}$ or $\mathbf{Y}_{M}$ since one do not know the Schwarz kernel of these compositions. That is why we proceed in several steps: one knows $\mathbf{u} \in \mathrm{RT}$ (RT is well-known lower-order Raviart-Thomas space [28]) at the iteration $n$ and one wants to compute $\mathscr{Z} \mathbf{u}$.

- First, we determine two potentials $\left(\phi_{\mathbf{u}}, \psi_{\mathbf{u}}\right)$ of $\mathbf{u}$ such that $\mathbf{u} \approx \mathbf{n} \times \nabla_{\Gamma} \psi_{\mathbf{u}}+\nabla_{\Gamma} \phi_{\mathbf{u}}$.

- We compute $\left(\phi_{\mathbf{J}_{u}}, \psi_{\mathbf{J}_{u}}\right)$ by solving (3.28) and we derive $\mathbf{J}_{u} \in \mathrm{RT} \approx \mathbf{n} \times \nabla_{\Gamma} \psi_{\mathbf{J}_{u}}+\nabla_{\Gamma} \phi_{\mathbf{J}_{u}}$.

- We compute $\mathbf{M}_{u}=\mathbf{u}-Z_{0} \eta \mathbf{n} \times \mathbf{J}_{u} \in \mathrm{RT}$.

- Finally, we compute the "classical" matrix-vector products coming from integral operators T and K.

In this section, we explain how we treat these four steps.

\subsection{First step}

First, recall some properties related to the Raviart-Thomas space [11]. Let $W_{h}$ be the subspace of RT defined by $W_{h}:=\left\{\mathbf{v} \in \mathrm{RT}: \nabla_{\Gamma} \cdot \mathbf{v}=0\right\}$. There is $C>0$ such that $\forall \mathbf{v} \in \mathrm{RT}$ verifying $\forall \mathbf{w} \in W_{h}(\mathbf{v}, \mathbf{w})=0$, the solution $p$ of $p \in H^{1}(\Gamma)^{\bullet}$ and $\Delta_{\Gamma} p=\nabla_{\Gamma} \cdot \mathbf{v}$ satisfies $\left|\mathbf{v}-\nabla_{\Gamma} p\right|_{0, \Gamma} \leq C h\left|\nabla_{\Gamma} \cdot \mathbf{v}\right|_{0, \Gamma}$. Moreover, each $\mathbf{v} \in \mathrm{RT}$ can be written in a unique way $\mathbf{v}=\mathbf{n} \times \nabla_{\Gamma} \psi+\mathbf{v}_{\perp}$, with $\psi \in \stackrel{o}{P_{1}}\left(\stackrel{o}{P_{1}}\right.$ denotes the space of scalar functions, piecewise linear on each triangle of the mesh and whose mean value is null on $\Gamma$ ) and $\mathbf{v}_{\perp}$ in the $L^{2}$-orthogonal of the kernel of the divergence operator in RT.

We begin by the computation of $\psi$ : for that it is sufficient to project $\mathbf{v}$ onto the space defined by:

$$
V_{\text {loop }}=\operatorname{span}\left\{\mathbf{n} \times \nabla_{\Gamma} \phi_{i}: i=1, \ldots, \text { nbnoe }-1\right\}
$$

where $\phi_{i}$ is the Lagrange basis function associated to the node $i$ of the mesh and nbnoe denotes the number of vertices. More precisely, we find $\mathbf{v}_{\text {loop }}=\sum_{i=1}^{n b n o e-1} \mathbf{v}_{\text {loop }}^{i} \mathbf{n} \times \nabla_{\Gamma} \phi_{i}$ such that $\forall \mathbf{w} \in V_{\text {loop }}$,

$$
\int_{\Gamma} \mathbf{v}_{\text {loop }} \cdot \mathbf{w} \mathrm{d} \Gamma=\int_{\Gamma} \mathbf{v} \cdot \mathbf{w} \mathrm{d} \Gamma
$$

Finally, we simply have $\psi=\sum_{i=1}^{n b n o e-1} \mathbf{v}_{\text {loop }}^{i} \phi_{i}$ where $\psi$ is obviously determined modulo one constant. We can remark that this computation only implies the inversion of a small sparse linear system whose $((n b n o e-1) \times$ $($ nbnoe - 1)).

Now we have to determine a potential $\phi \in \stackrel{o}{P}$ such that $\mathbf{v}_{\perp} \approx \nabla_{\Gamma} \phi$. For that, we suggest to solve: find $\phi \in \stackrel{\circ}{P}$ such that $\forall \phi^{\prime} \in \stackrel{\circ}{P} 1$

$$
-\int_{\Gamma} \nabla_{\Gamma} \phi \cdot \nabla_{\Gamma} \phi^{\prime} \mathrm{d} \Gamma=\int_{\Gamma} \nabla_{\Gamma} \cdot \mathbf{v} \phi^{\prime} \mathrm{d} \Gamma=\int_{\Gamma} \nabla_{\Gamma} \cdot \mathbf{v}_{\perp} \phi^{\prime} \mathrm{d} \Gamma
$$

As $\forall \mathbf{w} \in W_{h}\left(\mathbf{v}_{\perp}, \mathbf{w}\right)_{0, \Gamma}=0, \phi$ solution of (5.3) is a finite element approximation of the problem: find $p \in H^{1}(\Gamma)^{\bullet}$ such that $\Delta_{\Gamma} p=\nabla_{\Gamma} \cdot \mathbf{v}=\nabla_{\Gamma} \cdot \mathbf{v}_{\perp}$. One easily deduces from it that the a priori error estimate $\left|\mathbf{v}_{\perp}-\nabla_{\Gamma} \phi\right|_{0, \Gamma} \leq C h\left|\nabla_{\Gamma} \cdot \mathbf{v}\right|_{0, \Gamma}$ holds.

In conclusion, the determination of $(\psi, \phi)$ requires the resolution of two sparse systems. We also use the MUltifrontal Massively Parallel sparse direct Solver (MUMPS) [26] to solve them. MUMPS provides a quick and low cost inversion. 


\subsection{Second step}

The system (3.28) can be written as two discrete weak formulations:

find $\left(\phi_{\mathbf{J}_{u}}, V_{j}, U\right) \in\left[P_{1}\left(\mathscr{T}_{h}\right)\right]^{2} \times \stackrel{\circ}{P}_{1}\left(\mathscr{T}_{h}\right)$ such that for all $\left(\phi^{\prime}, V_{j}^{\prime}, U^{\prime}\right) \in\left[P_{1}\left(\mathscr{T}_{h}\right)\right]^{2} \times \stackrel{\circ}{P}_{1}\left(\mathscr{T}_{h}\right)$

$$
\left\{\begin{aligned}
A_{0}\left(\phi_{\mathbf{J}_{u}}, \phi^{\prime}\right)-\sum_{j=1}^{p} \frac{A_{j}}{k_{\varepsilon}^{2}}\left(\nabla_{\Gamma} V_{j}, \nabla_{\Gamma} \phi^{\prime}\right)+\left(U, \phi^{\prime}\right) & =\frac{1}{Z_{0}}\left(\psi_{\mathbf{u}}, \phi^{\prime}\right) \\
\left(V_{j}, V_{j}^{\prime}\right)-\frac{B_{j}}{k_{\varepsilon}^{2}}\left(\nabla_{\Gamma} V_{j}, \nabla_{\Gamma} V_{j}^{\prime}\right)-\left(\phi_{\mathbf{J}_{u}}, V_{j}^{\prime}\right) & =0 \text { for all } j=1, \ldots, p \\
\left(\nabla_{\Gamma} U, \nabla_{\Gamma} U^{\prime}\right)+\left(\eta \mathbf{n} \times \nabla_{\Gamma} \phi_{\mathbf{J}_{u}}, \mathbf{n} \times \nabla_{\Gamma} U^{\prime}\right) & =0
\end{aligned}\right.
$$

and find $\left(\psi_{\mathbf{J}_{u}}, V_{j}, U\right) \in\left[P_{1}\left(\mathscr{T}_{h}\right)\right]^{2} \times \stackrel{\circ}{P}_{1}\left(\mathscr{T}_{h}\right)$ such that for all $\left(\psi^{\prime}, V_{j}^{\prime}, U^{\prime}\right) \in\left[P_{1}\left(\mathscr{T}_{h}\right)\right]^{2} \times \stackrel{\circ}{P}_{1}\left(\mathscr{T}_{h}\right)$

$$
\left\{\begin{aligned}
\left(\psi_{\mathbf{J}_{u}}, \psi^{\prime}\right)+A_{0}\left(U, \psi^{\prime}\right)-\sum_{j=1}^{p} \frac{A_{j}}{k_{\varepsilon}^{2}}\left(\nabla_{\Gamma} V_{j}, \nabla_{\Gamma} \psi^{\prime}\right) & =-\frac{1}{Z_{0}}\left(\left(1+\frac{\Delta_{\Gamma}}{k_{\varepsilon}^{2}}\right)^{\frac{1}{2}} \phi_{\mathbf{u}}, \psi^{\prime}\right) \\
\left(V_{j}, V_{j}^{\prime}\right)-\frac{B_{j}}{k_{\varepsilon}^{2}}\left(\nabla_{\Gamma} V_{j}, \nabla_{\Gamma} V_{j}^{\prime}\right)-\left(U, V_{j}^{\prime}\right) & =0 \text { for all } j=1, \ldots, p \\
-\left(\nabla_{\Gamma} U, \nabla_{\Gamma} U^{\prime}\right)+\left(\eta \nabla_{\Gamma} \psi_{\mathbf{J}_{u}}, \nabla_{\Gamma} U^{\prime}\right) & =0
\end{aligned}\right.
$$

where $(\cdot, \cdot)$ denotes the classical $L^{2}$-scalar product.

Remark 5.1. In (5.5), we also must determine the square root term $\left(\left(1+\frac{\Delta_{\Gamma}}{k^{2}}\right)^{\frac{1}{2}} \phi_{\mathbf{u}}, \psi^{\prime}\right)$. For that, we proceed in this way:

$$
\left(\left(1+\frac{\Delta_{\Gamma}}{k_{\varepsilon}^{2}}\right)^{\frac{1}{2}} \phi_{\mathbf{u}}, \psi^{\prime}\right) \approx A_{0}\left(\phi_{\mathbf{u}}, \psi^{\prime}\right)-\sum_{j=1}^{p} \frac{A_{j}}{k_{\varepsilon}^{2}}\left(\nabla_{\Gamma} V_{j}, \nabla_{\Gamma} \psi^{\prime}\right)
$$

where $V_{j} \in P_{1}\left(\mathscr{T}_{h}\right)(j=1, \ldots, p)$ is solution of the discrete weak formulation $\left(V_{j}, V_{j}^{\prime}\right)-\frac{B_{j}}{k_{\varepsilon}^{2}}\left(\nabla_{\Gamma} V_{j}, \nabla_{\Gamma} V_{j}^{\prime}\right)=$ $\left(\phi_{\mathbf{u}}, V_{j}^{\prime}\right)$ for all $V_{j}^{\prime} \in P_{1}\left(\mathscr{T}_{h}\right)$.

All these sparse linear systems are solved for by using MUMPS. The size of these systems is about $(2+p) n t r i / 2$ (with ntri is the number of triangle of the mesh and we have used the approximation nbnoe $\approx$ ntri/2). In the next section, we show that $p=2$ is a relevant choice. In this case, the size becomes $2 n t r i$. It is not a big system in the case of surface meshes and we have remarked [14] that the cost of the resolution of these kind of system by MUMPS is always negligible compared to the one of the resolution of the integral equation (dense system), even when we use a Fast Multipole Method.

Finally, $\mathbf{J}_{u} \in$ RT is obtained by a simple projection of $\mathbf{n} \times \nabla_{\Gamma} \psi_{\mathbf{J}_{u}}+\nabla_{\Gamma} \phi_{\mathbf{J}_{u}}$ on RT.

\subsection{Third step}

The determination of $\mathbf{M}_{u}$ is less obvious than one could believe. Indeed, the straightforward way to derive $\mathbf{M}_{u} \in \mathrm{RT}$ is to do a simple projection of $\mathbf{M}_{u}=\mathbf{u}-Z_{0} \eta \mathbf{n} \times \mathbf{J}_{u}$ on RT. But, it seems that the property proved in the previous section (i.e. $\mathscr{Z} \sim a \mathrm{Id}+$ Compact) is not "sufficiently" preserved after the discretization. To avoid this problem, it is more judicious to compute $\mathbf{M}_{u}$ by using $\mathbf{n} \times \nabla_{\Gamma} \psi_{\mathbf{u}}+\nabla_{\Gamma} \phi_{\mathbf{u}}$. Indeed, if $\eta$ is constant, one can see that $\mathbf{M}_{u} \approx \mathbf{n} \times \nabla_{\Gamma}\left(\psi_{\mathbf{u}}-Z_{0} \eta \phi_{\mathbf{J}_{u}}\right)+\nabla_{\Gamma}\left(\phi_{\mathbf{u}}+Z_{0} \eta \psi_{\mathbf{J}_{u}}\right)$ and $\mathbf{Y}_{M}$ is better closed on the continuous operator $\mathbf{Y}_{M}^{e x}$ than when we directly use $\mathbf{u}$. 
This choice has a direct impact on the solutions. Indeed, let us look how the impedance condition is verified to the discrete level: $\forall \mathbf{w}_{h} \in \mathrm{RT}$, we have

$$
\begin{aligned}
\left(\mathbf{E}^{h} \times \mathbf{n}+Z_{0} \eta \mathbf{n} \times\left(\mathbf{H}^{h} \times \mathbf{n}\right), \mathbf{w}_{h}\right) & =\left(\mathbf{g}+\frac{1}{2}\left(-\mathbf{u}+\mathbf{M}_{u}+\eta Z_{0} \mathbf{n} \times \mathbf{J}_{u}\right), \mathbf{w}_{h}\right) \\
& =\left(\mathbf{g}+\frac{1}{2}\left(-\mathbf{u}+\mathbf{n} \times \nabla_{\Gamma} \psi_{\mathbf{u}}+\nabla_{\Gamma} \phi_{\mathbf{u}}-Z_{0} \eta \mathbf{n} \times \mathbf{J}_{u}+Z_{0} \eta \mathbf{n} \times \mathbf{J}_{u}\right), \mathbf{w}_{h}\right) \\
& =\left(\mathbf{g}+\frac{1}{2}\left(-\mathbf{n} \times \nabla_{\Gamma} \psi_{\mathbf{u}}-\mathbf{u}_{\perp}+\mathbf{n} \times \nabla_{\Gamma} \psi_{\mathbf{u}}+\nabla_{\Gamma} \phi_{\mathbf{u}}\right), \mathbf{w}_{h}\right) \\
& =\left(\mathbf{g}+\frac{1}{2}\left(\nabla_{\Gamma} \phi_{\mathbf{u}}-\mathbf{u}_{\perp}\right), \mathbf{w}_{h}\right)
\end{aligned}
$$

We can make some remarks:

- For the continuous case, the space $\mathbf{H}_{\times}^{-1 / 2}\left(\operatorname{div}_{\Gamma}, \Gamma\right)$ admits a hodge decomposition and we have $\mathbf{u}_{\perp}=\nabla_{\Gamma} \phi_{\mathbf{u}}$.

- For the discrete case, if one choose $\mathbf{M}_{u}=\mathbf{u}-Z_{0} \eta \mathbf{n} \times \mathbf{J}_{u}$, then the boundary condition is verified in this classical sense:

$$
\left(\mathbf{E}^{h} \times \mathbf{n}+Z_{0} \eta \mathbf{n} \times\left(\mathbf{H}^{h} \times \mathbf{n}\right), \mathbf{w}_{h}\right)=\left(\mathbf{g}, \mathbf{w}_{h}\right), \quad \forall \mathbf{w}_{h} \in \mathrm{RT}
$$

whereas by using our choice $\mathbf{M}_{u}=\mathbf{n} \times \nabla_{\Gamma} \psi_{\mathbf{u}}+\nabla_{\Gamma} \phi_{\mathbf{u}}-Z_{0} \eta \mathbf{n} \times \mathbf{J}_{u}$, (5.7) leads to

$$
\sup _{\mathbf{w}_{h} \in \mathrm{RT}} \frac{\left(\mathbf{E}_{h} \times \mathbf{n}+Z_{0} \eta \mathbf{n} \times\left(\mathbf{H}_{h} \times \mathbf{n}\right)-\mathbf{g}, \mathbf{w}_{h}\right)_{0, \Gamma}}{\left\|\mathbf{w}_{h}\right\|_{0, \Gamma}} \leq \frac{1}{2}\left\|\mathbf{u}_{\perp}-\nabla_{\Gamma} \phi_{\mathbf{u}}\right\|_{0, \Gamma} \leq C h\left|\nabla_{\Gamma} \cdot \mathbf{u}\right|_{0, \Gamma}
$$

since the Raviart-Thomas space does not subject to an exact Helmholtz decomposition. Nevertheless, the numerical results will show that this perturbation is under control.

\subsection{Fourth step}

The discretization of the operators $\mathrm{T}$ and $\mathrm{K}$ by the Raviart-Thomas finite elements is well-known and that is why we do not detail this here. What is less obvious is the computation of $\mathbf{n} \times \mathrm{T}$. We have already treated this difficulty in [14] when we wanted to construct a Combined Field Integral Equation for the impedance problems. The technique is based on a discrete analogue of the Helmholtz decomposition and has been proposed by Christiansen and Nédélec in [12]. For the completeness of the paper, we briefly describe it in this paper:

Let $l_{h}$ be a linear form defined on RT; the problem is to construct some $\Theta^{h} l_{h}$ that mimics $\mathbf{n} \times l_{h}$. In what follows, $P_{0}^{o}$ denotes the space of scalar functions whose restriction to any triangle is constant and whose mean value is null on $\Gamma$ and $P_{1}^{o}$ denotes the space of scalar functions, piecewise linear on each triangle of the mesh and whose mean value is null on $\Gamma$. We consider the following saddle-point problem:

$$
\begin{aligned}
& \text { Find }\left(u^{h}, q^{h}\right) \in \mathrm{RT} \times P_{0}^{o} \text { such that for all }\left(u^{\prime}, q^{\prime}\right) \in \mathrm{RT} \times P_{0}^{o}, \\
& \qquad \begin{array}{ll}
\int_{\Gamma} u^{h} \cdot u^{\prime} \mathrm{d} \Gamma+\int_{\Gamma} q^{h} \operatorname{div} u^{\prime} \mathrm{d} \Gamma & =l^{h}\left(u^{\prime}\right) \\
\int_{\Gamma} q^{\prime} \operatorname{div} u^{h} \mathrm{~d} \Gamma & =
\end{array}
\end{aligned}
$$

Once this system is solved, we associate to $\left(u^{h}, q^{h}\right)$ the following element of RT:

$$
v^{h}=\Theta^{h} l^{h}=\mathscr{P}_{\mathrm{RT}}\left(u^{h} \times \mathbf{n}\right)-\operatorname{curl} \mathscr{P}_{P_{1}^{o}}\left(q^{h}\right),
$$

where $\mathscr{P}_{X}$ denotes the $\mathrm{L}^{2}$-projection on the finite element space $X$. 
The map $\Theta^{h}$ is analyzed in [12] and we have improved its determination by using the Loop-Star basis functions [20].

Now, we are going to explain how to reduce the cost of the (dense) matrix-vector products. There are normally four vector products to form the two terms: $T 1=K \mathbf{M}_{u}+i Z_{0} T \mathbf{J}_{u}$ and $T 2=i Z_{0} K \mathbf{J}_{u}+T \mathbf{M}_{u}$. We remark that if one computes the two quantities $Q 1=(K+T)\left(\mathbf{M}_{u}+i Z_{0} \mathbf{J}_{u}\right)$ and $Q 2=(K-T)\left(\mathbf{M}_{u}-i Z_{0} \mathbf{J}_{u}\right)$, we simply have $T 1=(Q 1+Q 2) / 2$ and $T 2=(Q 1-Q 2) / 2$ i.e. only two matrix-vector products. Moreover [14] proposed an algorithm which only uses two fast multipole calculations to determine $K \mathbf{u}+T \mathbf{v}$ and $K \mathbf{v}+T \mathbf{u}$.

The last steps are:

- Calculation of $\Theta^{h}\left(K \mathbf{M}_{u}+i Z_{0} T \mathbf{J}_{u}\right) \approx \mathbf{n} \times\left(K \mathbf{M}_{u}+i Z_{0} T \mathbf{J}_{u}\right)$ : [14] proved that this step is negligible compared to the (dense) matrix-vector products even when FMM is used.

- Calculation of $i \eta\left[i Z_{0} K \mathbf{J}_{u}+T \mathbf{M}_{u}\right]$ : first we compute $\mathbf{v} \in \mathrm{RT} \approx\left[i Z_{0} K \mathbf{J}_{u}+T \mathbf{M}_{u}\right]$ by using a sparse inversion of the RT mass matrix (i.e. we make a simple projection of this term on the RT finite elements space) and then we form the sparse matrix-vector product $\left(i \eta \mathbf{v}, \mathbf{v}^{\prime}\right)$ with $\mathbf{v}^{\prime} \in \mathrm{RT}$.

\section{Numerical RESUlts}

The aim of this part is to numerically prove the good iterative behavior of the method. We will also show that the solutions are accurate. Each result will be compared to the one obtained by using a Impedance Combined Field Equation (ICFIE) that we have proposed in [14]. We have proved that ICFIE is a relevant method to treat constant and variable impedance problems. The goal of this first study is to show that it is possible to construct a cheap naturally well-conditioned formulation for the partially coated object and to give a first numerical issue. The GMRES solver [19] is used to solve for the linear system. In the following, $\Gamma_{m}$ and $\Gamma_{i}$ correspond respectively to parts of $\Gamma$ where the impedance operator is equal or not to zero.

We consider four scattering objects illuminated by a plane wave with a wavenumber $k$ :

- A sphere (smooth surface) of diameter $1 \mathrm{~m}$. The meshes are composed of 1500, 6000 and 13500 edges which respectively correspond to wavenumbers $k$ equal to 4.83, 11 and 16.4. The discretization complies with the criterion of around 10 points per wavelength. We have simulated two situations: the first is a constant impedance $\eta=0.34$ i.e. $\Gamma_{m}=\emptyset$ and the second is defined by $\eta=0.34$ on $\Gamma_{i}=\{(x, y, z)$ : $x^{2}+y^{2}+z^{2}=1$ and $\left.z>0\right\}$.

- A cube (non-smooth surface). The meshes are composed of 2178, 4860, 8460 and 13374 edges which respectively correspond to wavenumbers $k$ equal to $10,15,20$ and 25 . We have also considered two situations: the first is a constant impedance $\eta=0.34$ and for the second $\eta=1$ on $\Gamma_{i}$ which corresponds to a face of the cube. The incident plane wave goes on a corner of the cube where the material is discontinuous.

- A gap-toothed cube (non-convex and non-smooth surface). The mesh is composed of 20628 edges and the wavenumber $k$ is equal to $14 \mathrm{~m}^{-1}$. $\Gamma_{i}$ is composed of three faces as depicted in Figure 1. On each face, we impose a different impedance value (piecewise constant impedance). In particular, we have taken $\eta=0.3,0.5,0.8$.

- A Channel cavity (non-convex, non-smooth surface and cavity effect) which is depicted in Figure 2. The mesh is composed of 153033 edges and the wavenumber $k$ is equal to $105 \mathrm{~m}^{-1}$. We take $\eta=$ $0.035+0.035 i$ on $\Gamma_{i}$ which corresponds to the circular part to the bottom of the cavity. For this example, we have coupled the ICFIE with a SParse Approximate Inverse (SPAI) preconditioner.

First, we focus on the influence of $p$ (i.e. the truncation of the Padé expansion) on the convergence of iterative solver and the accuracy of the solution. In [16], it is proved that $p=8$ and $\theta=\pi / 3$ yield to a satisfactory approximation of the square-root operator. These values are derived in order to construct an efficient On-surface Radiation Condition (OSRC) in the high-frequency domain. So, the degree of the approximation has a direct impact on the accuracy of the solution. In our case, this impact can be qualified of "indirect" (in comparison of the previous) in the sense that it is firstly the integral formulation (3.17) which is modified and then it is its discretization which gives the quality of the solution. So, one can have an accurate solution for $p=0$. 


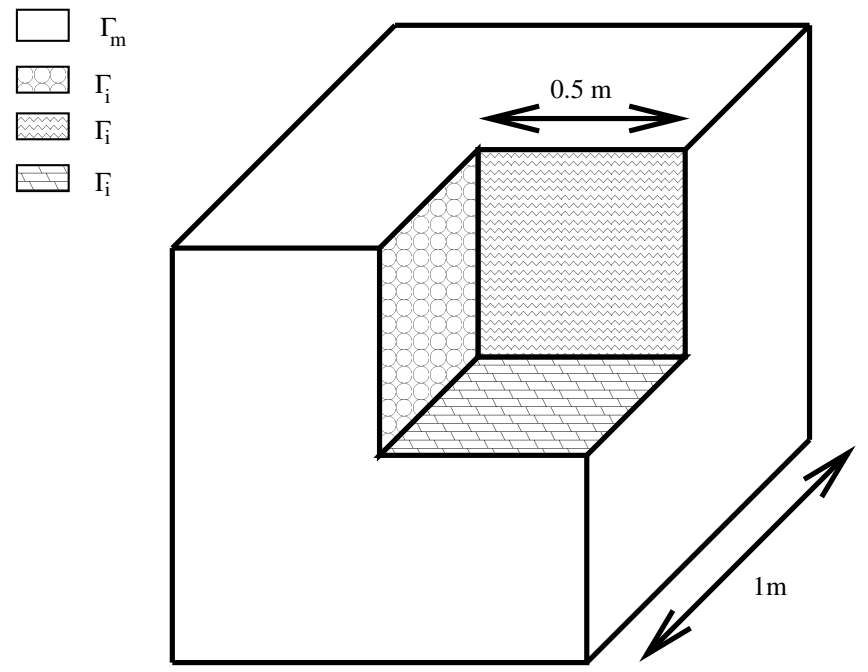

Figure 1. Gap-toothed cube.

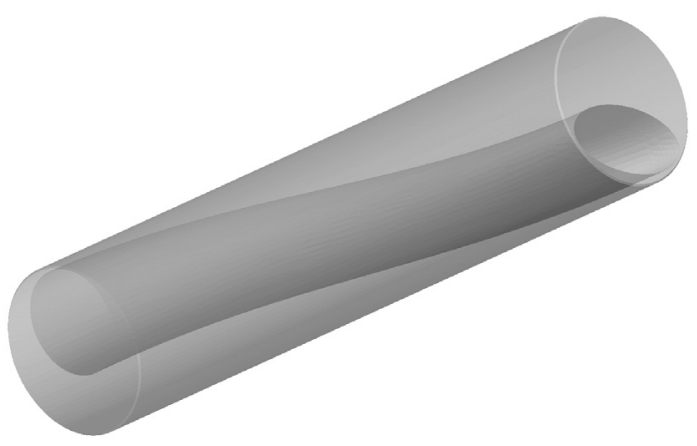

Figure 2. Channel cavity.

We clarify this point in order to avoid all confusion. One thus can hope to find a compromise between the robustness (number of iterations) and the accuracy. To illustrate that, we consider the case of the partially coated cube with 8460 edges and $k=20$. Figure 4 represents the Radar Cross Section (RCS) in function of $p$. We can see that the solutions are similar. With regards to the robustness, Figure 3 shows that for this configuration the number of iterations reaches its minimum ( 25 for a residual equals to $\left.10^{-8}\right)$ as soon as $p=2$. For the rest of the numerical experiments, we have taken $p=2$.

Figures 7 give the RCS diagrams calculated with the new formulation and ICFIE for the box and the cavity. It shows that the accuracy of the proposed formulation is comparable to the one of ICFIE for RCS computation.

Figures 5 respectively show the evolution of the number of iterations according to the discretization (number of points per wavelength) and the wavenumber $k$ for the totally and partially coated sphere. For the new formulation, the number of matrix-vector product is independent of the discretization step and the frequency. Only 9 and 10 iterations are necessary to obtain the solution. For the ICFIE, one can observe a linear dependence which is most important for the space refinement. One also sees that the discontinuity of the material has a direct impact on the number of iterations for the two formulations. 


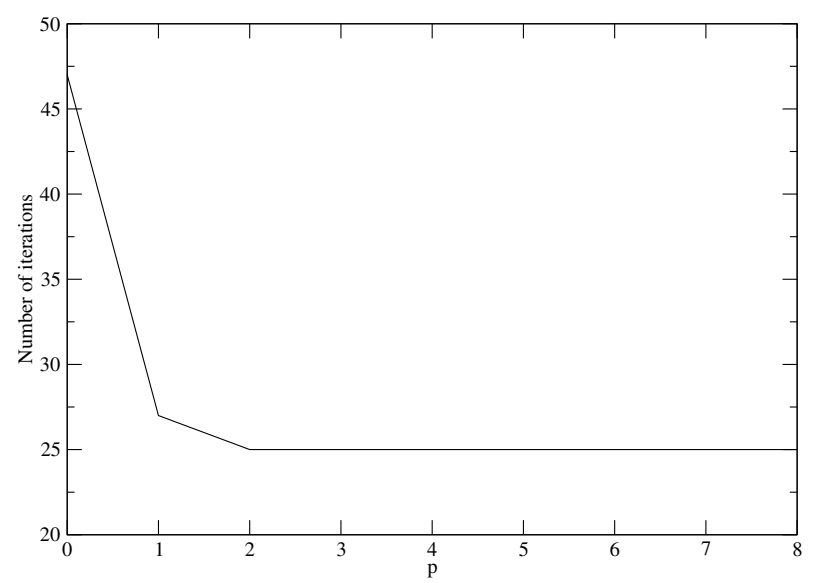

Figure 3. Number of iterations in function of $p$ for the partially coated cube, $k=20$ and the residue equals to $10^{-8}$.

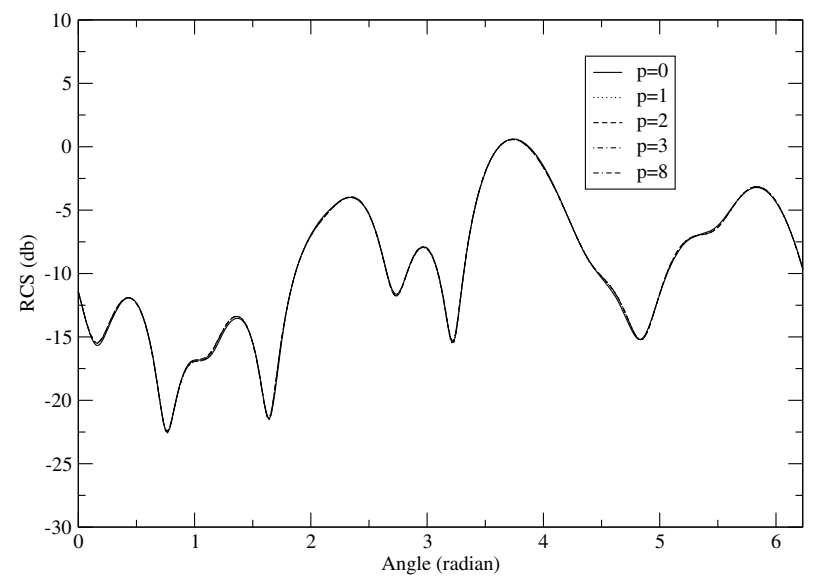

FIGURE 4. RCS in function of $p$ for the partially coated cube, $k=20$ and the residue equals to $10^{-8}$.
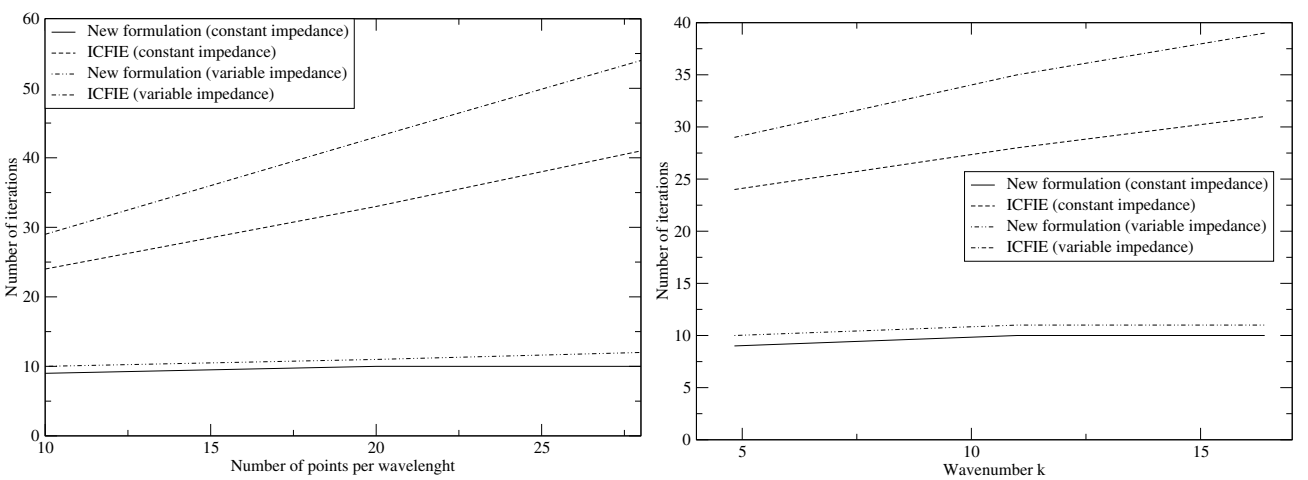

Figure 5. Behavior for the space refinement (left-hand side) and the frequency increase (righthand side) for the sphere. 

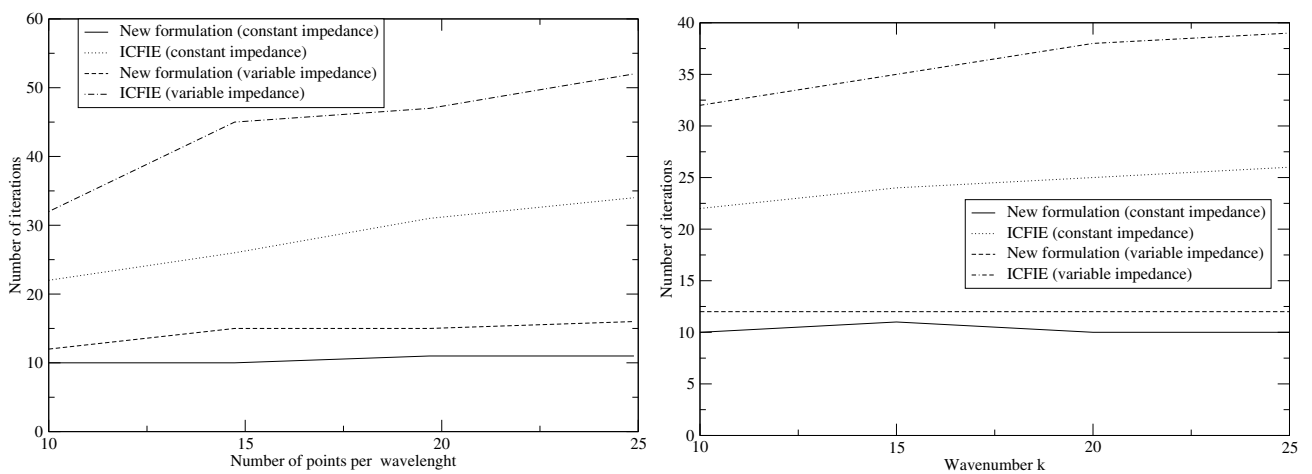

FiguRE 6. Behavior for the space refinement (left-hand side) and the frequency increase (righthand side) for the cube.
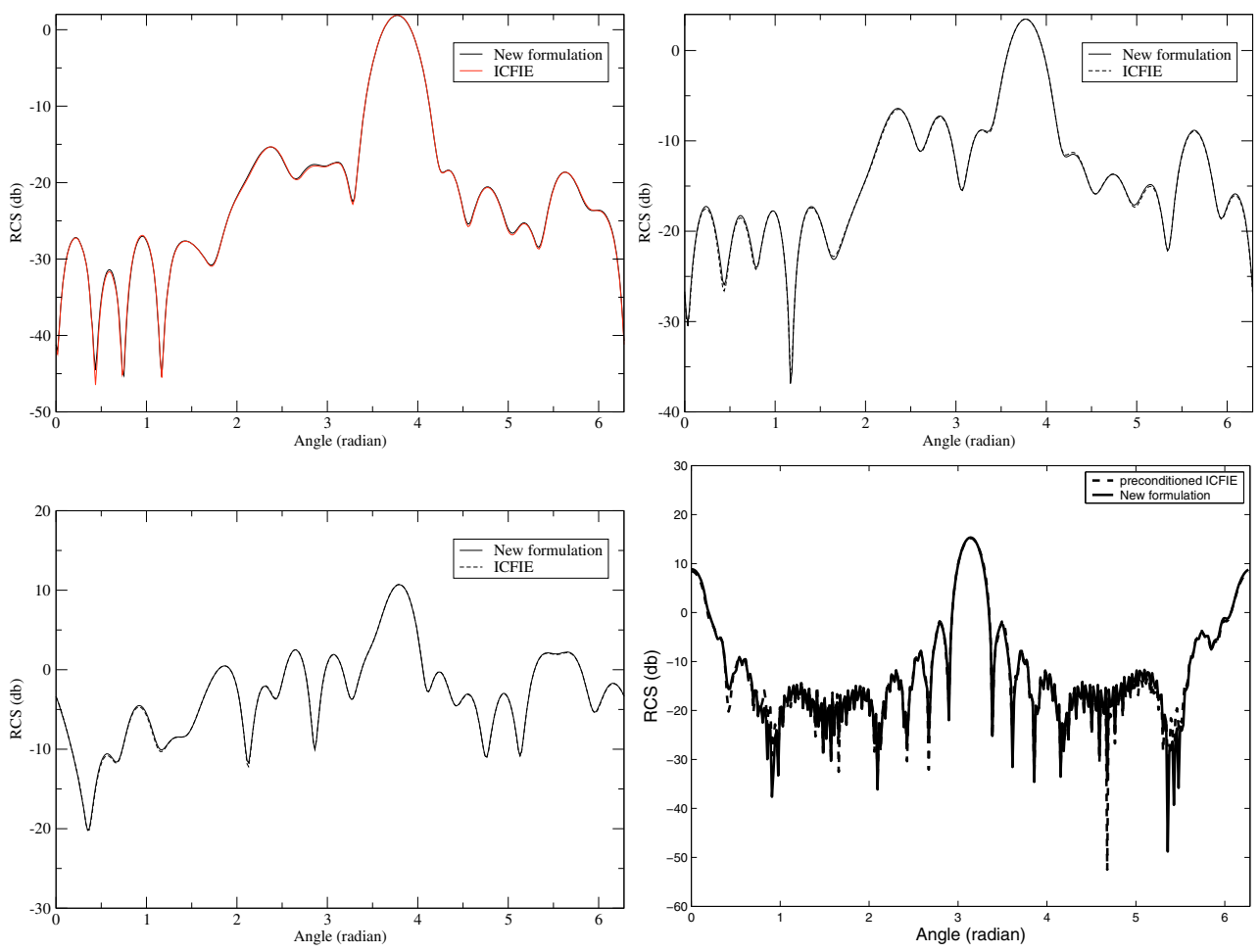

FiguRE 7. Comparison of the RCS between the new formulation and the ICFIE for the cube (up) (13 374 edges and $k=25)$ with constant (left-hand side) and variable (right-hand side) impedance, the gap-toothed cube (down-left-hand side) and the channel cavity (down-righthand side).

We have carried out the same analysis for the cube. The results are given in Figures 6. For ICFIE, the conclusion is the same one as for the sphere. For the new formulation, we observe a slight discretization dependence. Nevertheless, the stability of the method stays good and the gain in terms of iteration is still important.

Finally, we have tested the method for two partially coated non-convex geometries: the gap-toothed cube and the channel cavity. Figure 8 shows the evolution of residues according to the number of matrix-vector products 

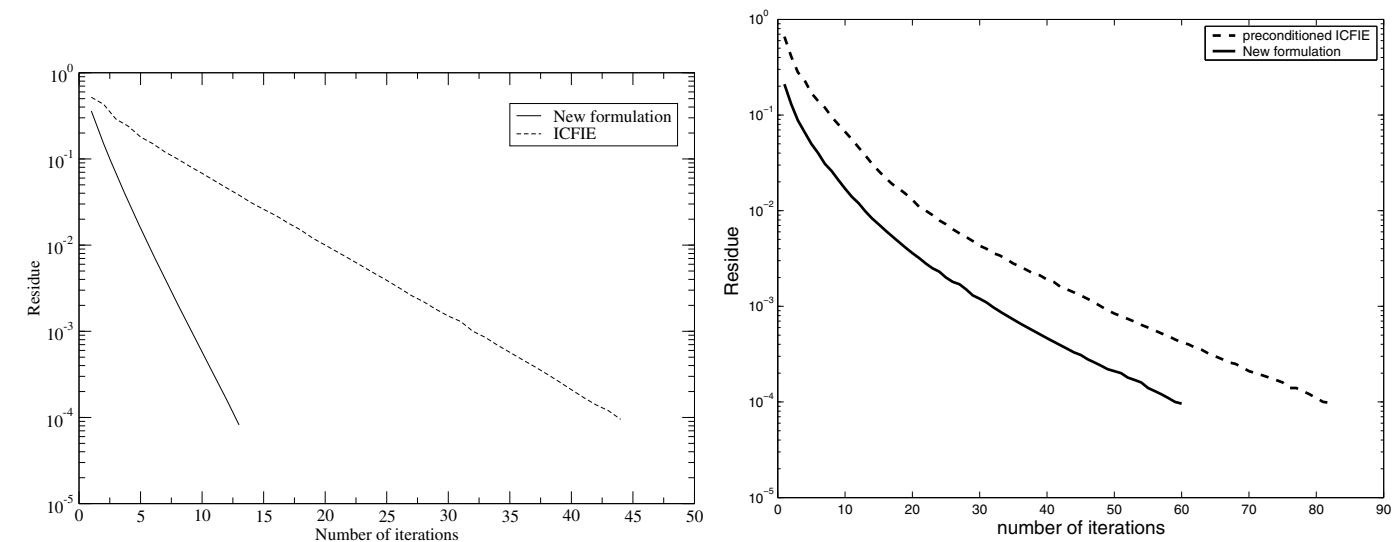

FIgURE 8. Evolution of the residue according to the number of matrix-vector products for the gap-toothed cube (left-hand side) and the channel cavity (right-hand side).

for a GMRES tolerance equals to $10^{-4}$. We observe an good convergence rate for these types of geometry. With concern the channel cavity, we have used a SPAI preconditioner for the ICFIE and we have speed up the CPU time of a factor 3 by using the new formulation.

\section{Conclusion}

We have proposed a well-conditioned integral formulation for the electromagnetic scattering with the variable Leontovitch condition. Several numerical test-cases have proved that this formulation leads to convergence rates (for a GMRES solver) significantly better than the one of ICFIE. Moreover, these rates are almost frequency and discretization-step independent. The accuracy of solutions (for the RCS) are of the same order than ICFIE. Finally, this method seems to be a good candidate to efficiently treat more complicated problems (in particular a greater number of degrees of freedom). Indeed, as we have already said, it only requires two fast multipole calculations whereas we use four of this one for ICFIE. Moreover, the choice of $p=2$ for the truncation of the Padé expansion implies small sparse matrices that one can treat at lower cost by MUMPS. This study is actually in progress. A comparison with ICFIE preconditioned by a SPAI will be interesting to really evaluate the cost of the method.

\section{REFERENCES}

[1] F. Alouges, S. Borel and D. Levadoux, A stable well conditioned integral equation for electromagnetism scattering. J. Comput. Appl. Math. 204 (2007) 440-451.

[2] X. Antoine and H. Barucq, Microlocal diagonalization of strictly hyperbolic pseudodifferential systems and application to the design of radiation conditions in electromagnetism. SIAM J. Appl. Math. 61 (2001) 1877-1905.

[3] X. Antoine and M. Darbas, Generalized combined field integral equations for the iterative solution of the three-dimensional Helmholtz equation. ESAIM: M2AN 41 (2007) 147-167.

[4] A. Bendali, M'B Fares and J. Gay, A boundary-element solution of the Leontovitch problem. IEEE Trans. Antennas Propagat. 47 (1999) 1597-1605.

[5] Y. Boubendir, Techniques de décomposition de domaine et méthode d'équations intégrales. Ph.D. Thesis, INSA, France (2002).

[6] A. Buffa, Hodge decomposition on the boundary of a polyhedron: the multi-connected case. Math. Mod. Meth. Appl. Sci. 11 (2001) 1491-1504.

[7] A. Buffa and R. Hiptmair, Galerkin Boundary Element Methods for Electromagnetic Scattering, in Computational Methods in Wave Propagation, M. Ainsworth, P. Davies, D.B. Duncan, P.A. Martin and B. Rynne Eds., Lecture Notes in Computational Science and Engineering 31, Springer-Verlag (2003) 83-124.

[8] F. Cakoni, D. Colton and P. Monk, The electromagnetic inverse scattering problem for partially coated Lipschitz domains. Proc. Royal. Soc. Edinburgh 134A (2004) 661-682. 
[9] S.L. Campbell, I.C.F. Ipsen, C.T. Kelley, C.D. Meyer and Z.Q. Xue, Convergence estimates for solution of integral equations with GMRES. Tech. Report CRSC-TR95-13, North Carolina State University, Center for Research in Scientific Computation, USA (1995).

[10] S.L. Campbell, I.C.F. Ipsen, C.T. Kelley and C.D. Meyer, GMRES and the Minimal Polynomial. BIT Numerical Mathematics 36 (1996) 664-675.

[11] H.S. Christiansen, Résolution des équations intégrales pour la diffraction d'ondes acoustiques et électromagnétiques - Stabilisation d'algorithmes itératifs et aspects de l'analyse numérique. Ph.D. Thesis, Centre de Mathématiques Appliquées, UMR 7641, CNRS/École polytechnique, France (2002).

[12] S. Christiansen and J.C. Nédélec, A preconditioner for the electric field integral equation based on Calderon formulas. SIAM J. Numer. Anal. 40 (2002) 1100-1135.

[13] F. Collino, S. Ghanemi and P. Joly, Domain decomposition method for the Helmholtz equation: a general presentation. Comput. Methods Appl. Mech. Eng. 184 (2000) 171-211.

[14] F. Collino, F. Millot and S. Pernet, Boundary-integral methods for iterative solution of scattering problems with variable impedance surface condition. PIER 80 (2008) 1-28.

[15] D. Colton and R. Kress, Inverse acoustic and electromagnetic scattering theory, Applied Mathematical Sciences 93. Springer, Berlin, Germany (1992).

[16] M. Darbas, Préconditionneurs analytiques de type Calderon pour les formulations intégrales des problèmes de direction d'ondes. Ph.D. Thesis, INSA Toulouse, France (2004).

[17] M. Darbas, Generalized CFIE for the Iterative Solution of 3-D Maxwell Equations. Appl. Math. Lett. 19 (2006) $834-839$.

[18] M. Darbas, Some second-kind integral equations in electromagnetism. Preprint, Cahiers du Ceremade 2006-15 (2006) http://www. ceremade.dauphine.fr/preprints/CMD/2006-15.pdf.

[19] V. Frayssé, L. Giraud, S. Gratton and J. Langou, A Set of GMRES Routines for Real and Complex Arithmetics on High Performance Computers. CERFACS Technical Report, TR/PA/03/3 (2003) http://www.cerfacs.fr/algor/Softs/GMRES/ index.html.

[20] J.-F. Lee, R. Lee and R.J. Burkholder, Loop star basis functions and a robust preconditioner for EFIE scattering problems. IEEE Trans. Antennas Propagat. 51 (2003) 1855-1863.

[21] M.A. Leontovitch, Approximate boundary conditions for the electromagnetic field on the surface of a good conductor, Investigations Radiowave Propagation part II. Academy of Sciences, Moscow, Russia (1978).

[22] J.R. Mautz and R.F. Harrington, A combined-source solution for radiation and scattering from a perfectly conducting body. IEEE Trans. Antennas Propag. AP-27 (1979) 445-454.

[23] F.A. Milinazzo, C.A. Zala, G.H. Brooke, Rational square-root approximations for parabolic equation algorithms. J. Acoust. Soc. Am. 101 (1997) 760-766.

[24] K.M. Mitzner, Numerical solution of the exterior scattering problem at eigenfrequencies of the interior problem. Int. Scientific Radio Union Meeting, Boston, USA (1968).

[25] P. Monk, Finite Element Methods for Maxwell's Equations, Numerical Mathematics and Scientific Computation. Oxford Science Publication, UK (2003).

[26] Multifrontal Massively Parallel Solver, www.enseeiht.fr/lima/apo/MUMPS.

[27] J.C. Nédélec, Acoustic and Electromagnic Equations Integral Representation for Harmonic Problems. Springer, New York, USA (2001).

[28] S.M. Rao, D.R. Wilton and A.W. Glisson, Electromagnetic scattering by surfaces of arbitrary shape. IEEE Trans. Antennas Propagat. AP-30 (1982) 409-418.

[29] V. Rokhlin, Diagonal form of translation operators for the Helmholtz equation in three dimensions. Appl. Comput. Harmon. Anal. 1 (1993) 82-93.

[30] O. Steinbach and W.L. Wendland, The construction of some efficient preconditioners in the boundary element method. Adv. Comput. Math. 9 (1998) 191-216.

[31] B. Stupfel, A hybrid finite element and integral equation domain decomposition method for the solution of the 3-D scattering problem. J. Comput. Phys. 172 (2001) 451-471. 